\title{
Balkanologie
}

Balkanologie Revue d'études pluridisciplinaires

Vol. XIV, $\mathrm{n}^{\circ}$ 1-2 | 2012

Volume XIV Numéro 1-2

\section{La Bosnie-Herzégovine post-conflit. État(s) redistributeur(s)}

\section{Hugues Sachter}

\section{Q OpenEdition}

1 Journals

\section{Édition électronique}

URL : https://journals.openedition.org/balkanologie/2354

DOI : 10.4000/balkanologie.2354

ISSN : 1965-0582

Éditeur

Association française d'études sur les Balkans (Afebalk)

\section{Référence électronique}

Hugues Sachter, "La Bosnie-Herzégovine post-conflit. État(s) redistributeur(s) », Balkanologie [En ligne], Vol. XIV, nº 1-2 | 2012, mis en ligne le 08 février 2013, consulté le 28 juin 2022. URL : http:// journals.openedition.org/balkanologie/2354; DOI : https://doi.org/10.4000/balkanologie.2354

Ce document a été généré automatiquement le 17 décembre 2020.

(c) Tous droits réservés 


\title{
La Bosnie-Herzégovine post-conflit. État(s) redistributeur(s)
}

\author{
Hugues Sachter
}

1 La Bosnie-Herzégovine (plus loin $\mathrm{BiH}$ ) abandonne peu à peu les thèmes de la sortie de conflit (armes, réfugiés, reconstruction), mais des interrogations persistent sur la stabilité à long terme du pays, et sur la manière de lui assurer un avenir pérenne, autonome, et si possible européen. Cette option suppose une adaptation des solutions issues de l'accord de Dayton, qui fondent un pays divisé en deux entités : la Fédération (croato-musulmane) de Bosnie-Herzégovine (plus loin $\mathrm{FBiH}$ ) et une entité serbe, la Republika Srpska (plus loin RS). Du point de vue économique, l'hypothèse sous-jacente a été celle d'une coexistence entre des options liées à un schéma canonique de transition, et un schéma politique destiné à régler territorialement des différends sécuritaires, par l'obligation d'un marché unique, de monnaie et de relations extérieures unifiées, et d'un système fiscal coordonné. En fait, comme dans toute transition post-communiste, surtout quand il n'y a pas eu de " révolution de velours ", le politique et l'économique persistent à s'interpénétrer sous des modalités atypiques par rapport à la pratique des démocraties libérales occidentales ${ }^{1}$.

Officiellement, la $\mathrm{BiH}$ est un pays d'économie de marché, dans un environnement international ouvert et convertible. En raison de la dépendance du pays vis-à-vis des autorités internationales (PIC, FMI, Banque mondiale, UE), l'acquiescement (compliance) déclaratif par rapport aux exigences d'insertion dans un environnement de plus en plus mondialisé est rituel, mais associé de réserves plus ou moins explicites. On pourrait trouver des racines intellectuelles et culturelles multiples à l'autonomisme affirmé de l'entité serbe, ou à l'unitarisme plus ou moins affirmé du côté de l'entité croatomusulmane ${ }^{2}$. Les deux parties font facilement référence, l'une à l'univers russe oriental, l'autre à un tiers monde encore vécu comme forme économique autonome, mais l'une comme l'autre se situent en retrait, voire en crispation par rapport à un projet global à visées économistes.

3 Les thèmes de l'ouverture et de l'intégration à l'OMC et à l'UE sont fréquemment marqués d'un protectionnisme larvé (par rapport à l'importation) ou d'un aveu de non- 
compétitivité dans le domaine de la qualité (avancée des frontières de l'Union à la frontière croate), et, plus discrètement, de la crainte d'une élévation des barrières par rapport à d'anciens marchés (Russie pour la Republika Srpska, en raison de l'accord de libre échange Russie-Serbie; anciens pays non alignés pour la Fédération; exYougoslavie pour tous). On peut cependant remarquer que les milieux scientifiques ou syndicaux ont tendance à persister dans le registre de la critique du capitalisme, avec une certaine nostalgie pour « d'autres mondes possibles».

4 Mais d'autres thèmes bien moins romantiques reviennent de manière récurrente dans les deux entités, et il est net que nombre des faiblesses du temps de l'autogestion se maintiennent à la surface : illiquidité des entreprises et salaires non payés, liquidations judiciaires toujours reportées, livraisons interentreprises perturbées, arriérés de contributions et taxes, sans parler de grands projets d'infrastructures toujours reportés. Le principal thème "nouveau » est celui des privatisations, de leur gestion opaque et de leurs conséquences néfastes. On pourrait résumer en disant que beaucoup de dysfonctionnements anciens persistent, alors leurs manifestations sous de nouvelles formes semblent appeler à regretter l'ancien temps.

5 Ces thèmes recèlent une nostalgie, celle d'une région jeune, dynamique, industrielle, insérée dans l'ensemble yougoslave (avec des variations plutôt mineures sur le rôle de la Serbie dans cet ensemble). Le rôle du territoire bosniaque dans la résistance, la gloire mondiale d'Ivo Andrić, les Jo de Sarajevo : autant de thèmes récents réappropriés sous divers modes selon le lieu ou le groupe. Bien entendu, la grandeur historique du royaume médiéval et de son église schismatique jouent en arrière-plan, conjointement avec les vizirs bosniaques et le Dragon de Bosnie (Zmaj od Bosne), voire l'insurrection de Nevesinje, mais sans guère d'impact direct sur les thèmes économiques (bien que la richesse minière de l'ancien royaume constitue une référence mentale forte, comme ailleurs dans les Balkans).

6 Ces considérations si importantes dans le champ idéologique et politique semblent sans lien avec la situation économique actuelle du pays. Elles peuvent être traitées comme purement idéologiques, voire irrationnelles, ou plutôt comme simple légitimation de divers groupes exerçant le pouvoir ou y aspirant. Mais c'est également une référence qui fait rentrer les valeurs économiques dans une logique de grandeur et de justification au sens de Boltanski et Thévenot ${ }^{3}$. Rappelons que cet ouvrage fondamental de la théorie des conventions décrit un environnement institutionnel propre à assurer la coordination des décisions à partir de ce que les auteurs appellent des mondes différents. Il serait tout à fait tentant d'appliquer cette théorie à des situations conflictuelles comme celle de la BiH, bien que les auteurs aient eux-mêmes reconnu que la théorie trouve ses limites dans les situations de rapports de force.

7 Mais l'application des conventions supposerait qu'existe une convention de «bosnité ». Or, celle-ci est largement récusée en RS, voire démentie par de constantes initiatives destinées à affirmer une option confédérale. Or, si l'on examine la régulation économique en $\mathrm{BiH}$ dans ses deux entités, voire dans les cantons de la $\mathrm{FBiH}$, on constate que, tant sur le plan du cadre institutionnel que sur celui des structures économiques, les points communs sont plus importants que les divergences. La législation sur les sociétés, le droit du travail, le système fiscal, le régime de protection sociale ne divergent que sur des points mineurs. Le système douanier et de TVA est unitaire, de même que la fiscalité sur les entreprises et les particuliers ne présentent pas de différence frappante (flat tax dans les deux cas). 
8 Mais il y a plus : l'ensemble de la BiH hérite de structures productives du temps de l'ancienne Yougoslavie et, mis à part la disparition d'unités productives étroitement liées aux options politiques de l'ancien système socialiste fédéral, on ne constate pas de renouvellement radical du tissu productif.

9 En présence d'un blocage des mutations économiques, loin de soumettre la situation à une critique radicale de l'héritage socialiste, la nostalgie d'un âge d'or alimente les considérations, sous des modalités variées selon le sentiment d'appartenance communautaire. Mais, comme toute la politique ancienne est accusée de méfaits vis-àvis des communautés ethno-religieuses, c'est la nature qui devient un socle commun de référence, et en quelque sorte la convention « de la nature bosniaque » cimente espoirs, mais aussi conflits d'appropriation.

10 Un examen objectif du passé économique exclut une référence à un âge d'or socialiste du territoire bosniaque, bien que le problème bosniaque n'ait jamais été traité comme un enjeu en soi par le régime, satisfait de la solution fédérale. Or, dans la période récente, après une consolidation opérée à l'issue du conflit armé, les structures économiques qui se sont reconstituées présentent des similitudes avec celles du passé. De plus, malgré tous les conflits institutionnels, elles ne permettent pas de distinguer radicalement les deux entités. Les différences observables ne franchissent pas les limites que la géographie régionale permet habituellement de repérer. Les politiques économiques ne diffèrent pas sur des points essentiels (il n'y pas, dirait-on, de modèle RS ou de modèle $\mathrm{FBiH})$. Que recherchent alors les pouvoirs régionaux ? Ne feraient-ils que se cramponner à la maîtrise de ressources pour entretenir leur bureaucratie partisane? Le multipartisme exacerbé en FBiH n'est-il qu'une forme de lotizzazione, comme dans l'Italie ou l'Autriche de l'après-Deuxième Guerre mondiale, et le monopole d'un parti en RS un décalque de solutions appliquées dans de nombreuses contrées de la CEI ? Nous verrons qu'il y a en $\mathrm{BiH}$ une interpénétration originale entre système politique et régulation économique, qui tente en permanence de reproduire le fondement de tout système socialiste : la contrainte budgétaire molle ${ }^{4}$, et ce malgré une forte contrainte extérieure en direction d'une discipline financière : comité d'émission (currency board), FMI, ASA/UE.

11 C'est pourquoi il nous semble nécessaire d'interroger tout d'abord le rapport entre les structures productives actuelles et l'héritage des temps antérieurs. Puis il nous faudra prendre en considération le fonctionnement économique réel de la $\mathrm{BiH}$ et de ses deux entités. Il faudra en particulier rendre compte de la contradiction qui existe entre un système productif dont les principales composantes se sont contractées, et un niveau de consommation général très nettement amélioré. C'est ce qui nous permettra ensuite de revenir au système de gouvernance et à ses rapports à l'intégration internationale.

1.

\section{L'héritage yougoslave et les structures actuelles}

12 La situation de 1990, année qui précède l'irruption des conflits armés, sert souvent de référence. Ce repère temporel correspond à peu près à l'agenda de la transition (en fait, les rapports annuels sur la transition (Transition) de la Banque Européenne pour la Reconstruction et le Développement -BERD- prennent la date symbolique de 1989), même si cette date n'a pas un lien immédiat avec des événements politiques de type «chute du mur " peu repérables en ex-Yougoslavie. Mais la coïncidence entre amorce de la transition et guerre ${ }^{5}$ introduit également des confusions ${ }^{6}$. 
13 La référence à 1990 a sans doute un sens politique, mais n'en a guère sur le plan économique. L'idée qu'elle sous-entend, à savoir que la Bosnie et Herzégovine était un pays en plein succès sur la voie du développement (et de l'intégration interculturelle, interreligieuse ou interethnique $)^{7}$, symbolisé par les JO de 1984 à Sarajevo, relève sans doute largement de l'« avenir radieux » au sens de Zinoviev. Elle est largement associée au thème du socialisme, garant de l'avenir des peuples yougoslaves, thème réitéré inlassablement par le Parti/Ligue des Communistes, et dont la BiH aurait constitué l'exemple le plus probant.

14 L'éclatement post-communiste, dont seul le caractère "nationaliste » est la plupart du temps retenu, a par sa forme guerrière, souvent atroce, laissé sur les acteurs une impression de malheur irréparable, ce qui joue certainement dans la valeur attribuée au «bon vieux temps». Mais en réalité cet éclatement n'a pas seulement modifié les présupposés économiques, il a surtout bouleversé le rapport du pays à sa démographie. Dans une société qui était en cours d'achèvement de sa transition démographique, le conflit a provoqué un choc triple : par les pertes humaines (100 000 morts et disparus ${ }^{8}$, correspondant à trois ans d'accroissement démographique de l'époque), par la chute consécutive radicale de la natalité (qui précède dorénavant ses voisins d'Europe postsocialiste sur le chemin d'une croissance démographique zéro) et par l'immense flux de réfugiés, dorénavant devenus exilés.

15 Mais à bien y regarder, la BiH n'est pas seule à avoir subi des chocs économiques et sociaux'. L'Albanie de 1991 à 1997 (anomie et émigration de masse), la Bulgarie de 1989 à 1997, le Kosovo entre 1989 et 1999 (apartheid, guerre, répression, et émigration), la Croatie de 1991 à 1995 (conflit territorial interne), la Macédoine de 1992 à 2001 (conflits armés sporadiques, pressions économiques de ses voisins, embargos), voire la Serbie de 1991 à 2000 (conflits aux frontières, embargo, pressions militaires), ont subi pressions économiques, chocs et conflits, qui ont ébranlé les conditions de fonctionnement de leurs économies et modifié leurs dynamiques démographiques. Dans le cas de la $\mathrm{BiH}$, il est intéressant d'effectuer la comparaison avec la Macédoine ${ }^{10}$, unité fédérale yougoslave dont les caractéristiques économiques quantitatives étaient très proches de celles de la $\mathrm{BiH}$ (toutes deux à $65 \%$ du niveau moyen yougoslave en $1989^{11}$ ), et qui recèle comme elle des hétérogénéités ethno-religieuses très marquées (quoique sous des modalités passablement différentes ${ }^{12}$ ). Et pourtant, parties du même point de départ, sans véritable guerre en Macédoine, les deux pays se retrouvent quantitativement au même point d'arrivée ${ }^{13}$. À bien y regarder, la Serbie se retrouve au contraire relativement moins bien lotie à l'issue du processus: alors que $\mathrm{BiH}$ et Macédoine se trouvaient en 1990 à $65 \%$ du niveau moyen fédéral, la Serbie sans Kosovo mais avec Vojvodine (configuration actuelle) affichait une moyenne fédérale. Or, actuellement, sans le Kosovo, et malgré le potentiel de la Vojvodine, elle dépasse à peine ses deux voisines. Peut-être faut-il rappeler qu'en Macédoine, la tradition de la pečalba ${ }^{14}$, ajoutée aux conséquences démographiques des multiples guerres et redécoupages territoriaux qu'elle a subis jusqu'en 1948, font du peuple macédonien un peuple chez qui le traumatisme de l'exil a depuis longtemps été intériorisé alors que, on le verra, l'émigration de masse est tombée brutalement sur la Bosnie ${ }^{15}$.

16 La Bosnie-Herzégovine qui est réapparue en 1992 (concrètement en 1996), comme un pays nouveau dans la galaxie des pays en transition, était jusque-là une région yougoslave. Même si la BiH était bien repérable dans l'ensemble yougoslave au sein d'une une Yougoslavie socialiste, mais complètement décentralisée ${ }^{16}$, la discontinuité 
économique due à la guerre et au changement de régime de régulation semble totale. La BiH était, disait-on, une Yougoslavie en petit (ce dont témoignait son drapeau). Certes, mais sur le plan du développement on serait tenté de dire « une Yougoslavie en tout petit ». Si la BiH constituait le témoignage des réussites yougoslaves, il n'y a pas de raison qu'elle ne le soit pas de ses échecs.

En effet, la BiH yougoslave et autogestionnaire n'a pas vécu un développement fulgurant, bien qu'elle ait pu accéder à une intégration au mode de vie des économies développées, tel qu'on pouvait le concevoir dans l'immédiat après-Seconde Guerre mondiale. Il faut donc reconsidérer la thématique de la référence à l'année 1990. C'est pourquoi nous avons examiné en premier lieu l'évolution du PIB par habitant de la BiH par comparaison avec les États-Unis (qui restent la référence la plus stable sur le long terme) et les républiques voisines, Serbie - dont le niveau de développement était toujours approximativement la moyenne de la RSFY, et Macédoine, pays dont nous avons souligné l'intérêt d'une comparaison avec la BiH.

\section{BOX : Note méthodologique}

Le niveau actuel d'activité économique se situe - en termes de PIB brut, selon la BERD ${ }^{17}$ - à $85 \%$ du niveau de 1989. Mais comme la BERD compte en euros par rapport à un indice BERD des 30 pays européens, et en raison des évolutions ultérieures de l'euro, ce mode de calcul n'est pas selon nous le plus propice aux comparaisons dans le long terme. Les comparaisons en SPA, utilisées pour leur part par Eurostats, ne permettent pas d'appréhender la dimension productive, et excluent explicitement des comparaisons diachroniques. Nous avons donc préféré nous rapporter aux chiffres de l'ONU en dollars US courants, et en se rapportant aux États-Unis, ce qui assure une continuité. En procédant ainsi, nous arrivons à $100 \%$ du chiffre de 1990. Pour la période yougoslave, nous appliquons un coefficient de 0,65 au PIB/h national, tant pour la $\mathrm{BiH}$ que pour la Macédoine. Lydall a quant à lui utilisé un coefficient de 0,85 , mais il voulait appréhender les SPA à l'intérieur de la Fédération yougoslave ${ }^{18}$.

Les résultats obtenus sont exposés dans le tableau 1.

Tableau 1. PIB/habitant en US \$ prix courants

\begin{tabular}{|c|c|c|c|c|c|c|c|c|}
\hline & Etats Unis & RSFY & $\mathrm{BiH}$ & $\mathrm{BiH} / \mathrm{USA}$ (\%) & SRB & $\mathrm{BiH} / \mathrm{SRB}$ (\%) & MK & MK / BiH (\%) \\
\hline 1970 & 4892 & 740 & 481 & 9,8 & 740 & 65 & 503 & 104,5 \\
\hline 1975 & 7409 & 1622 & 1054 & 14,2 & 1622 & 64,9 & 1102 & 104,6 \\
\hline 1980 & 12049 & 3263 & 2113 & 17,5 & 2251 & 93,8 & 1351 & 63,9 \\
\hline 1990 & 22716 & 3481 & 2262 & 9,9 & 2379 & 95,08 & 2344 & 103,8 \\
\hline 2001 & 35840 & & 1543 & 4,3 & 1523 & 101,3 & 1705 & 110,5 \\
\hline 2005 & 42330 & & 2885 & 6,8 & 3391 & 85,07 & 2937 & 101,8 \\
\hline
\end{tabular}




\begin{tabular}{|l|l|l|l|l|l|l|l|l|}
\hline $\mathbf{2 0 1 0}$ & 46546 & & 4478 & 9,6 & 5123 & 87,4 & 4434 & 99,01 \\
\hline
\end{tabular}

Source: United Nations National accounts main database, en ligne : unstats.un.org/unsd/snaama et SGJ 1980, 199019

19 L'examen de ces données confirme en apparence l'expression courante en $\mathrm{BiH}$ : « nous voici renvoyés aux années $1970 »$. Cependant, cette idée laisse de côté deux faits essentiels : en premier lieu, 1970 était proche d'un sommet (1975), à partir duquel les difficultés se sont accumulées pour l'ensemble de la Fédération yougoslave, pour aboutir à une décennie 1980 de crise continue et de croissance économique minimale ; en second lieu, la position économique de la BiH ne s'est jamais décisivement améliorée dans le contexte yougoslave. Le souci permanent de la question du Kosovo, et l'importance symbolique (et stratégique, on le verra) pour le régime de la coexistence ethnico-religieuse en $\mathrm{BiH}$ ont fait passer à l'arrière-plan la question quantitative de l'économie de la région.

20 La constitution de la configuration de l'économie bosniaque a résulté de la politique, sinon coloniale, tout au moins impérialiste, menée par l'Autriche-Hongrie de 1878 à 1918. Cette configuration s'est trouvée confortée pendant la Royauté, avec des influences étrangères diverses. Le régime socialiste a pour sa part enregistré l'acquis, pour lui faire suivre des évolutions dans le cadre d'une politique générale de la Fédération.

1. 1.

\subsection{Le développement sous le socialisme, une dépendance structurelle d'ensemble}

21 Sous l'influence des initiatives prises par l'Autriche-Hongrie lors de sa période d'occupation (1878-1918), et consécutivement aux années de crise de l'entre-deuxguerres, la situation dont héritait la Yougoslavie socialiste en BiH était assez classique pour un pays « du Sud ». Il s'agissait d'une économie duale avec de grandes entreprises lourdes très technologiques, et un environnement pauvre et de culture rurale traditionnelle (basse productivité, natalité élevée et alphabétisation basse). Les intérêts autrichiens avaient créé un nombre important de sites industriels liés aux ressources naturelles (minerais, bois, énergie), de sorte qu'il existe peu de sites d'industrie de base (à l'exception de l'aluminium de Mostar) qui ne remontent pas à l'ère autrichienne ${ }^{20}$.

Dans la première période de projets industriels socialistes de type stalinien, et dans un contexte centralisé (1947-1960), la BiH offrait une base industrielle pour un démarrage de l'accumulation. Par ailleurs, dans une région où s'était déroulée la guerre des Partisans, la diffusion d'une modernité (obligatoirement industrielle dans l'air du temps) était un impératif primordial, d'autant que la ressource humaine qualifiée faisait défaut pour l'industrie.

Après 1953, et surtout après 1960, les voies du développement de la République fédérée de BiH ont pris d'autres chemins: si les rentes naturelles fournissaient des intrants pour les filières aval, la promotion au niveau yougoslave d'ensemble d'un socialisme «de marché » a suscité la promotion d'« import substitutions » dans le domaine des biens de consommation, avec une certaine division spatiale des tâches. Mais les moyens financiers de la croissance yougoslave ont été assurés par l'obtention de dons (puis de crédits internationaux à bas taux) pour financer les investissements massifs qui y 
étaient associés, en jouant sur la position clé de la Yougoslavie dans le champ des relations Est-Ouest ${ }^{21}$. La crédibilité de cette position était liée à l'affichage d'un haut taux d'indépendance militaire, qui reposait sur une armée dotée de capacités défensives élevées et si possible d'origine nationale, mais aussi de capacités de résistance diffuse du niveau civil sur le terrain, réminiscence de la guerre des partisans : cela impliquait, outre une organisation de l'industrie de défense, le retour aux principes de Foča (du nom des ordonnances sur la vie économique en territoire libéré édictées dès 1942 par Moše Pijade ${ }^{22}$ ), fondées sur l'autosuffisance territoriale en cas de morcellement du pays sous un choc. À une ouverture internationale valorisant par exemple le tourisme pour financer les investissements, était associée une volonté quasi autarcique locale pour les biens de première nécessité (avec sans doute un secret espoir de voir les revenus générés sur le territoire dépensés sur place, pour améliorer les conditions de financement du circuit bancaire ${ }^{23}$ ). Cette position a été confortée par les options prises par la Ligue des Communistes à la suite de la crise pétrolière ${ }^{24}$ en faveur d'un développement des ressources locales.

La BiH s'est trouvée au cœur de cette politique : dotée de ressources exportables, voire exportatrice nette (même si ces ressources étaient de peu de valeur unitaire, et étaient en outre dépendantes des marchés internationaux), elle participait à la couverture des importations indispensables à l'édification des projets industriels. Comme elle constituait la région la plus à l'abri d'une invasion et donc propice à la localisation des productions militaires, tout en devant préserver des conditions de vie relativement autonomes, elle s'imposait comme point d'implantation des industries militaires. La BiH était donc contrainte à être intégrée dans un projet d'ampleur nationale, ce qui l'a quelque peu située en marge du projet central de développement des biens de consommation.

De ce fait, l'alimentaire, handicapé par la situation défavorable globale de la région visà-vis des productions végétales ${ }^{25}$, et plus paradoxalement le textile, n'ont aucunement connu le développement qui aurait pu accompagner une première phase d'industrialisation (en contrepoint par rapport à la Macédoine). Par contre, quand le complexe économique militaire (INVOJ), s'est mis en quête d'un équilibre économique autonome ${ }^{26}$, l'économie bosniaque s'est à nouveau, comme en 1946, retrouvée au cœur d'un projet d'intérêt national. La BiH s'est donc trouvée en situation dominée dans les rapports de filière, fournisseur d'intrants ${ }^{27}$, fournisseur de devises pour le système fédéral, et enfin génératrice de crédibilité pour le système militaire, exerçant un contrôle étroit depuis Belgrade (associé à une domination technologique pour la plupart des produits). Les exceptions majeures à ce schéma furent des aventures personnelles, celles de l'ingénierie des réseaux électriques (Emerik Blum et Energoinvest, puis Aluminij Mostar) et l'industrie de la volaille (Fikret Abdić et Agrokomerc) qui mettent surtout en évidence, par les dimensions personnelles des deux hommes, l'absence d'entrepreneuriat collectif local.

1. 1.

\subsection{Une pression démographique et un impératif d'intégration sociale}

L'après-guerre mondiale et la première phase de pouvoir socialiste ont généré un important mouvement en matière d'éducation et de santé. L'amélioration des conditions sanitaires a donné lieu à une dynamique démographique (baisse de la mortalité infantile, baisse de la mortalité naturelle) à laquelle ni le développement industriel ni le développement rural n'étaient prêts à répondre. Si la transition 
démographique s'est opérée au point qu'elle était quasiment achevée en 1990, l'effet des cohortes précédentes n'avait pas fini de faire sentir son effet sur le marché de l'emploi. De 1948 à 1991, la part de la population de BiH en Yougoslavie est passée de 16,3\% (2 564000 hbts) à 18,6\% (4 366000 hbts), avec une cassure à compter de 1971 $(18,3 \%)^{28}$. La croissance annuelle revenait de 62500 à 42100 personnes. La transition se perçoit dans le passage de la natalité de 37,3\% en 1955 à 14,1 \% en 1990. Cette évolution s'est répercutée sur la taille des ménages : 498000 ménages en 1948 (5,15 h./ménage) et 1203172 ménages en 1991 (3,63 h./ménage).

Le marché du travail s'est trouvé hors d'état d'absorber un tel flux, principalement parce que les structures économiques de la BiH n'avaient pas été conçues à cette fin. Le solde des emplois créés dans le secteur socialisé (celui donc qui investit) depuis 1963, et de la population en âge de travailler depuis 1963 ont été les suivants :

Tableau 2. Flux de population et d'emplois, stock de chômeurs 1963-1990

\begin{tabular}{|l|l|l|l|l|}
\hline & $\begin{array}{l}\text { Flux de population en âge de } \\
\text { travailler } \\
(>15 \text { ans })\end{array}$ & $\begin{array}{l}\text { Flux d'emplois créés } \\
\text { net des départs en } \\
\text { retraite }\end{array}$ & \multicolumn{2}{l|}{$\begin{array}{l}\text { Demandeurs } \\
\text { d'emploi } \\
\text { (Stock) }\end{array}$} \\
\hline $1963-1970$ & 254.000 & 58.201 & $(1970)$ & 37.998 \\
\hline $1971-1980$ & 815.000 & 309.408 & $(1980)$ & 136.865 \\
\hline $1981-1990$ & 357.000 & 205.418 & $(1990)$ & 283.478 \\
\hline Total & $1,426.000$ & 573.027 & & \\
\hline
\end{tabular}

Sources : tableaux 201-6, 201-7, 201-9 in SGJ $1990^{29}$

On trouve donc un déficit d'emplois offerts de 862573 personnes. Il ne faut pas s'étonner si le nombre de chômeurs déclarés, qui approchait les 100000 en 1975, avait fini par s'élever à 283478 en 1990. Deux voies se sont donc offertes : 1) maintien en surnombre en milieu rural et 2) émigration, interne, puis externe.

29 1) La solution de maintien de la population rurale sur place a fait partie d'une politique rurale générale de la Yougoslavie ${ }^{30}$, qui n'a pas donné priorité à la productivité par extension des exploitations ${ }^{31}$, mais qui a ainsi trouvé un mode de recrutement industriel peu coûteux ${ }^{32}$. Le niveau d'urbanisation s'en est trouvé limité (36,2\% de taux d'urbanisation pour $17,3 \%$ de population agricole en 1981), ce qui se répercute dans la situation actuelle.

2) L'émigration a été institutionnelle d'abord (recolonisation des terres «allemandes » en Vojvodine, 50000 personnes $^{33}$, puis est devenue émigration de travail, d'abord dans les républiques plus développées, et enfin à l'étranger dans le mouvement général amorcé dans les années 1960. De la sorte, le solde migratoire interne révèle un déficit de 489135 personnes $^{34}$, le plus élevé dans un pays où les mobilités inter-républicaines étaient très faibles. L'émigration internationale a pris le relais, atteignant presque 200000 personnes (140 000 travailleurs) en $1991^{35}$. Il faut noter que les migrations ont sans doute suivi des lignes communautaires ${ }^{36}$, les musulmans étant peu présents dans les migrations internes (87400 sur 1760000 migrations totales dans la RSFY 
1945-1981) ${ }^{37}$. Il convient donc de garder à l'esprit qu'en 1990, en additionnant les émigrés internes de 1945 à 1981 aux migrants temporaires à l'étranger, 550000 personnes vivaient déjà en dehors de leur BiH d'origine.

Par contre, et ce n'est pas contradictoire avec les remarques précédentes, la $\mathrm{BiH}$ a fait l'objet d'un grand effort d'intégration culturelle au monde moderne ${ }^{38}$, tout au moins tel qu'on l'entendait dans un régime socialiste jusqu'aux années 1970. La santé, l'éducation (alphabétisation, puis scolarisation), ont fait des progrès considérables (malgré un taux d'analphabétisme resté élevé en 1990, de 14,5\% de la population, particulièrement chez les femmes, 23,3\% en 1981, ce qui n'est pas si éloigné du Kosovo, respectivement 17,6 \% et $25,4 \%$ ). À la suite de la scolarisation des femmes, ces dernières ont accédé à l'emploi formel (32,1\% en 1981, y compris agriculture $\left.{ }^{39}\right)$, ce qui a bouleversé leur statut de genre, et sans doute contribué, conjointement à la scolarisation, à la transition démographique.

En conclusion, on constate que la BiH a moins généré d'emplois que la pression démographique ne l'aurait exigé, mais que le chômage a été amorti par la mobilité de la population, ce qu'a facilité l'effort culturel entrepris à la Libération. En effet, à l'intégration technique de l'économie avec les républiques plus développées s'est ajoutée la possibilité d'intégration individuelle, facteur de mobilité, ce qui est l'un des plus grands contrastes que l'on puisse observer par rapport au Kosovo. C'est peut-être aussi l'une des raisons d'un relatif sentiment de quiétude des instances yougoslaves face à la situation économique préoccupante de la République fédérée de BiH.

1. 1.

\subsection{Les structures productives et le système fédéral}

Si l'on examine la dynamique de la croissance et des emplois jusqu'en 1990, et plus particulièrement dans la décennie de crise 1980-1990, on trouve une explication à la nostalgie, alors qu'objectivement la situation était tout à fait insatisfaisante.

Il y a eu dans la décennie 1980-1990 des glissements propres à l'économie bosniaque par rapport à la Fédération dans son ensemble. En effet, après l'envolée des années 1970, qui avait mis en évidence des distorsions structurelles spécifiques au système autogestionnaire ${ }^{40}$, ces distorsions ont littéralement grippé le développement du pays ${ }^{41}$. Alors que la production (à prix constants 1972) avait cru de 1965 à 1980 à des taux dépassant les $5 \%$ annuels, le rythme de croissance s'est radicalement réduit dans la décennie 1980, passant à 0,7\% dans le premier quinquennat (baisse de 1,5\%/ an de la consommation; Lyall mentionne une chute de $25 \%$ des salaires réels dans le secteur productif ${ }^{42}$ ), pour se réduire à $0,3 \%$ dans la fin de la décennie (augmentation de $0,5 \% / a n$ de la consommation) ${ }^{43}$.

La BiH ne pouvait échapper à cette tendance générale, même si son système productif a amélioré sa position relative de 1979 à 1989 , passant de $12,18 \%$ de la production nationale à $12,8 \%^{44}$, avec une croissance de $12,8 \%$ sur dix ans (ce qui ne fait qu'un modeste $1,21 \%$ par an néanmoins) ${ }^{45}$. Simultanément l'emploi a ostensiblement cru de $28,6 \%^{46}$ à un rythme de 2,3\% par an ${ }^{47}$. Dans le même temps, les services non marchands subissaient des restrictions, conduisant à des augmentions mesurées de l'emploi, et surtout à des compressions de salaires ${ }^{48}$.

Les chiffres de l'emploi, une fois décomposés par branches (cf. tab. 3), font néanmoins apparaître des distorsions entre production et emploi, surtout lorsqu'on se rapporte aux productions matérielles concernées. En résumé, mis à part la volonté d'alléger la 
pression sur le marché du travail, il semble bien que les statistiques cachent des nondits sur la place de l'industrie militaire dans la république fédérée de $\mathrm{BiH}$ : la production de bicyclettes et de machines à écrire, explicitement citée comme retombée du complexe militaire ${ }^{49}$, ne présentait pas une dynamique suffisante pour justifier le niveau d'emploi dans les industries mécaniques ( $49 \%$ d'augmentation de l'emploi en $\mathrm{BiH}$ sur la décennie, mais $116 \%$ pour les machines et $120 \%$ pour les moyens de transport) $)^{50}$, voire dans la transformation des produits chimiques $(+64 \%$ ). Ceci renvoie aux observations de V. Bojičić qui attribue à la BiH $50 \%$ des capacités militaires de la Fédération yougoslave ${ }^{51}$. Il faut ajouter que le modèle adopté pour la BiH ne faisait pas la part belle aux biens de consommation quotidiens : textile-habillement (néanmoins $81 \%$ de croissance d'emploi) et industries agro-alimentaires ( $144 \%$ de croissance) $)^{52}$ se situaient au niveau minimal requis pour assurer la consommation locale : c'était la fameuse fermeture républicaine tant décriée ${ }^{53}$, mais indispensable à une autarcie locale indissociable du modèle géopolitique garant du financement des investissements (voir plus haut) $)^{5}$.

Par contre, s'il est un point sur lequel la BiH se distinguait des autres unités fédérales, c'était la faiblesse de son agriculture, surtout dans le domaine céréalier, et à l'inverse son importance dans le domaine (clé en ces temps de crise des échanges) des exportations (la $\mathrm{BiH}$ était structurellement excédentaire en terme de commerce extérieur) ${ }^{55}$. Si l'on admet l'hypothèse militaire, il faut également avoir en tête que l'armement était devenu un secteur exportateur (13\% des exportations yougoslaves en 1987) et qu'il échappait sans doute largement aux limitations de devises pour ses investissements, cette conjoncture se reflétant sur l'économie bosniaque ${ }^{56}$.

L'organisation industrielle présentait une autre spécificité donnant une apparence de puissance, les "giganti ». Ces ensembles, (en jargon autogestionnaire «SOUR », složena organizacija udruženog rada, organisation complexe du travail associél, étroitement associées au système bancaire, organisaient une intégration horizontale ${ }^{57}$ de branche au niveau de la République fédérée. Ce phénomène, présent dans toutes les républiques yougoslaves, était particulièrement accentué en BiH. Elles concentraient les branches de la région: UPI (agro alimentaire), UNIS (mécanique), Energoinvest (ingénierie et électricité de puissance), Krivaj (bois), EP (produits pétroliers), HEPOK (vins), etc., formant des holdings de fait, concentrant les moyens financiers ${ }^{58}$.

Une mention particulière revient au secteur agro-industriel qui, au travers des PIK (poljoprivredni industrijski kombinat, combinat agro-industriel), contrôlaient à la fois la collecte des produits agricoles et leurs processus de transformation. $\mathrm{Si}$, dans la plupart des cas, les combinats avaient un caractère sous-régional, en BiH le système UPI, qui comprenait également une banque (qui a survécu : UPI Banka), couvrait l'ensemble de la république fédérée, et intégrait un réseau de distribution alimentaire. L'économie rurale de toute la BiH était donc solidement quadrillée.

En fait, rien n'indique que les "giganti » aient constitué des entités économiques dotées d'une stratégie industrielle qui auraient pu conduire à des centres de profit lors des privatisations. Il s'agissait en fait d'entités de gestion des politiques de l'État actionnaire, organisant les compromis entre Fédération et République fédérée, assortissant projet d'État (armement, export, import substitution) et considérations clientélistes locales (emploi, rapports ville-campagne). Dans un contexte d'économie de pénurie (au sens de J. Kornai), elles permettaient de mobiliser les masses financières nécessaires aux investissements tout en maintenant un voile d'incertitude quant à la 
partie militaire des complexes (compte tenu des difficultés croissantes de liquidités, le système des grands groupes permettait d'organiser les transferts de fonds indispensables à l'exécution minimale des paiements).

Il ne faut donc pas s'étonner si, en définitive, les groupes ont éclaté sous l'effondrement de l'encadrement politique de l'économie, comme dans d'autres pays en transition. Par contre, c'est la manière dont a été géré l'éclatement (bien que l'asset stripping ${ }^{59} \mathrm{n}$ 'ait pas concerné que la $\mathrm{BiH}$ ), sous l'effet de rapports de situation de guerre et de clientélisme politique, qui laisse planer le doute sur la légitimité de ces restructurations. Ce sont en général des initiatives managériales (Zenica et sa sidérurgie ${ }^{60}$ ), associées au milieu politique local, qui ont permis le maintien d'un socle d'activités productives matérielles. Que ces activités aient en général dû leur survie à la disponibilité de ressources naturelles (et donc à l'existence de marchés tout organisés) contribue à renforcer la croyance en la nature comme socle de la richesse bosniaque.

1.

\title{
2. Les structures historiques vingt ans après, l'héritage et les mutations
}

\author{
1.
}

\subsection{La démographie, aubaine statistique, mutation traumatisante}

Les données macroéconomiques entre 1990 et 2010 sont difficilement comparables, ce qui tient à une hétérogénéité due au bouleversement démographique opéré dans le cours de la guerre 1992-1995 et dans sa suite immédiate. En effet, les comparaisons inter-temporelles s'effectuent habituellement en s'appuyant sur des hypothèses de dynamique démographique continue. Or, dans le cas bosniaque, on se trouve en présence d'une double, voire d'une triple discontinuité : les pertes humaines de la guerre, l'émigration/exil des réfugiés, l'effondrement de la natalité.

La population officiellement évaluée de la BiH, en attendant un recensement en 2013 (le premier depuis 1991), est de 3843000 habitants, à rapporter aux 4377000 habitants de 1991. Visiblement, la Fédération et la RS ne comptent pas de la même manière, puisque cette dernière affiche un chiffre net, tandis que la première distingue population résidente et population à l'étranger (probablement ceux qui sont partis comme réfugiés ${ }^{61}$ ). De ce fait, l'Agence des statistiques de la FBiH (FZS BiH) décompte pour sa part 527887 personnes "réfugiées à l'étranger». Cela implique une perte nette de 534000 personnes, ce qui, compte tenu des approximations, semble cohérent, bien qu'il faille tenir compte des 100000 victimes de guerre, pour $75 \%$ des hommes en âge de travailler ${ }^{62}$. Mais les évaluations récentes, tant de la Banque mondiale que des Ministères de la sécurité et du Ministère des réfugiés bosniaques, font apparaître une émigration permanente bien supérieure $(1350000)^{63}$, en incluant les enfants nés à l'étranger. Sur les neuf principaux pays d'accueil, ce sont 791348 personnes nées en BiH qui étaient recensées.

Mais tous les émigrés bosniaques ne sont pas des réfugiés de guerre : il y a les migrants dans les anciennes républiques yougoslaves qui incluent les migrants internes d'avant 1992 ( $c f$. plus haut), y compris en Slovénie - où une partie des immigrants bosniaques étaient en fait avant 1991 travailleurs en Slovénie tout en conservant leur statut de résidant en $\mathrm{BiH}$ - soit 490870 personnes et qui, d'une manière ou de l'autre prolongent une tendance de long terme. Il y a les émigrés économiques en UE d'avant 1992, au 
nombre de 200 000. Mais à l'inverse, un certain nombre de populations déstabilisées de la Krajina croate dans le sillage d'oluja se sont transplantées en RS.

La saignée démographique est d'autant plus manifeste que les pertes de la période de guerre correspondent à environ trois ans d'accroissement démographique des années 1980. Or, ce déficit s'est cumulé avec l'effondrement de la démographie interne à partir de 1998 : la natalité, qui avait modérément repris depuis 1998, a diminué durablement depuis 2000 (de 12,3 p.m. à 9 p.m.), pendant que la mortalité est montée lentement de 30000 à 35000 personnes par an, malgré un allongement progressif de la durée de vie (augmentée de 5 ans depuis 1998). Il en résulte une stagnation, voire un solde démographique négatif ${ }^{64}$.

Le résultat en est une difficulté à comparer les données économiques d'avant la partition par rapport à la situation d'après-guerre. L'amélioration de l'image économique représentée par le PIB/tête résulte en grande partie d'un effet purement statistique: la production se réfère à une population et à un nombre de ménages réduits (nombre de ménages revenu à 1054613 au lieu de 1208000 en 1990) par rapport à la période antérieure. Mais derrière cette image statique favorable se dissimulent des handicaps dynamiques déjà connus dans les régions de l'Europe du Sud qui ont subi une chute radicale de la natalité (manque d'offre de main d'œuvre qualifiée, charge des retraités et des personnes âgées, rationalité des réseaux d'infrastructure).

La richesse relative retrouvée vers le milieu des années 2000 n'est-elle qu'une illusion liée à l'émigration et à la stagnation démographique?

1. 1.

\subsection{Mutation des branches, stabilité des entités}

La comparaison 1980/1990/2010 met en évidence permanences et mutations dans le système productif, mais elle interroge sur les conditions nouvelles dans lesquelles elles fonctionnent. La partition yougoslave représente-t-elle la racine du mal, ou la mauvaise gestion du système économique issu de ce processus?

C'est pourquoi, dans un premier temps, nous avons mis en regard l'évolution d'avant 1990 (comparaison 1980, en fait 1979, et 1990) en termes de valeur ajoutée par branche (tab. 3) et d'emploi salarié vis-à-vis de la situation actuelle (tab. 4). Nous avons dans la suite tenté de comparer la situation dans les deux entités principales, FBiH, et RS, dans la situation actuelle ${ }^{65}$ (tab. 5).

50 Le mode de comptabilisation des activités ayant changé depuis la fin du socialisme, un reclassement des activités a dû être opéré. Tout d'abord, le Produit social (društveni proizvod) n'incorporait que la valeur ajoutée de la production matérielle et les services marchands, à la différence des comptabilités occidentales, ce qui a une influence sur l'appréciation globale concernant la place actuelle des services dans le pays ${ }^{66}$. Une seconde différence tient à certains reclassements de secteurs; nous avons pour cela suivi K. Peračković, et nous avons reconfiguré le découpage en secteurs par rapport à la normalisation actuelle ( $c f$. BOX $)^{67}$.

51 Ces tableaux mettent en évidence la désindustrialisation relative de la BiH actuelle, sur laquelle on reviendra. Mais l'image d'une complète tertiarisation doit être modérée par deux observations: on doit prendre en considération en premier lieu que les grands services publics liés au développement humain - santé et éducation - ont peu ou prou maintenu leurs effectifs (mais pas forcément leur soutenabilité financière ${ }^{68}$ ). En second 
lieu, bien des services marchands, surtout dans le domaine des services rendus aux entreprises, étaient au temps du socialisme internalisés dans les grandes firmes, vieux travers socialiste et plus encore autogestionnaire, afin d'y contrôler l'emploi tertiaire supérieur, la technologie et les investissements.

Il sort du cadre de ce travail de procéder à une analyse industrielle de la $\mathrm{BiH}$ branche par branche, et nous souhaitons principalement expliciter les grandes mutations en place relative. On perçoit que l'industrie est restée, avec le commerce, le principal producteur de valeur et pourvoyeur d'emplois dans le pays. Mais surtout, si on la regroupe avec les mines et l'énergie comme c'était le cas en comptabilité socialiste, et en ajoutant l'agriculture/sylviculture et le bâtiment génie civil pour trouver l'ensemble de la production matérielle, on découvre une forte réduction en termes de valeur (de $69,26 \%$ à 49,35\%), mais non pas un effondrement total. Par contre, il faut avoir en perspective que nombre d'activités de transformation se situent en première phase aval des activités d'exploitation des ressources (conserveries, scieries, alumine, acier brut, laminoirs, etc.), et que par conséquent l'activité de production de biens finaux a été radicalement réduite. Le système productif s'est donc dissocié de la consommation des ménages (et tout autant sans doute des biens d'équipement), ce qui implique une consommation reposant sur des importations.

Si l'on considère l'emploi en parallèle à la production de richesses, on constate la faiblesse relative de la productivité industrielle $(14,03 \%$ de la valeur ajoutée pour $19,43 \%$ des emplois). Ceci confirme les informations éclatées que l'on reçoit au quotidien quant à la mauvaise santé de l'industrie et au stockage d'emploi qui s'y opère au détriment de la profitabilité.

Tableau 3. VA BiH 1979,1989, 2010

\begin{tabular}{|c|c|c|c|c|c|c|c|}
\hline & $\begin{array}{c}\text { VA } \\
\text { marchande } \\
1979 \\
\text { (millions } \\
d Y U)\end{array}$ & $\begin{array}{c}\text { \% de VA } \\
\text { marchande } \\
\text { (1979) }\end{array}$ & $\begin{array}{c}\text { VA } \\
\text { marchande } \\
1989 \\
\text { (millions } \\
d Y U)\end{array}$ & $\begin{array}{c}\text { \% de VA } \\
\text { marchande } \\
\text { (1990) }\end{array}$ & $\begin{array}{c}\text { VA } \\
\text { totale } \\
2010 \\
\text { (milliers } \\
\text { KM) }\end{array}$ & $\begin{array}{c}\text { \% de VA } \\
\text { marchande } \\
2010\end{array}$ & $\begin{array}{l}\% \text { de } \\
\text { VA } \\
\text { totale }\end{array}$ \\
\hline $\begin{array}{l}\text { VA totale } \\
\text { (moins rente } \\
\text { imputée) }\end{array}$ & & & & & 19646992 & & $100 \%$ \\
\hline $\begin{array}{l}\text { total non } \\
\text { marchand }\end{array}$ & & & & & 5118074 & & 26,05 \\
\hline $\begin{array}{l}\text { L. } \\
\text { Administration }\end{array}$ & & & & & 2274239 & & \\
\hline M. Éducation & & & & & 1181219 & & \\
\hline
\end{tabular}




\begin{tabular}{|c|c|c|c|c|c|c|c|}
\hline $\begin{array}{l}\text { N. Sanitaire et } \\
\text { social }\end{array}$ & & & & & 1135058 & & \\
\hline $\begin{array}{l}\text { O. Communal et } \\
\text { social }\end{array}$ & & & & & 527558 & & \\
\hline total marchand & 151493 & 100 & 27738000 & 100 & 14528918 & $100 \%$ & 73,95 \\
\hline $\begin{array}{l}\text { total prod } \\
\text { matérielle }\end{array}$ & 95006 & 62,71 & 19211000 & 69,26 & 7170420 & 49,35 & 36,50 \\
\hline $\begin{array}{l}\mathrm{A}+\mathrm{B} \text {. } \\
\text { Agriculture, } \\
\text { forêts, pêche }\end{array}$ & 19729 & 13,02 & 2701000 & 9,74 & 1739818 & $11,97 \%$ & 8,86 \\
\hline C. Mines & 6159 & 4,07 & 1173000 & 4,23 & 491701 & $3,38 \%$ & 2,50 \\
\hline $\begin{array}{l}\text { D. Ind. de } \\
\text { transformation }\end{array}$ & 45718 & 30,18 & 12063000 & 43,49 & 2757317 & $18,98 \%$ & 14,03 \\
\hline E. Énergie & 4798 & 3,17 & 1349000 & 4,86 & 1098903 & $7,56 \%$ & 5,59 \\
\hline F. BGC & 18602 & 12,28 & 1925000 & 6,94 & 1082681 & $7,45 \%$ & 5,51 \\
\hline $\begin{array}{l}\text { total services } \\
\text { marchands }\end{array}$ & 41339 & 27,29 & 8749000 & 31,54 & 7358038 & 50,64 & 37,45 \\
\hline $\begin{array}{l}\text { G. Commerce, } \\
\text { services à la } \\
\text { personne }\end{array}$ & 22798 & 15,05 & 5091000 & 18,35 & 3242704 & $22,32 \%$ & 16,50 \\
\hline H. Hôtellerie & 3084 & 2,04 & 614000 & $2,21 \%$ & 491149 & $3,38 \%$ & 2,50 \\
\hline $\begin{array}{l}\text { I. Transports, } \\
\text { communications }\end{array}$ & 9780 & 6,46 & 1743000 & 6,28 & 1698298 & $11,69 \%$ & 8,64 \\
\hline $\begin{array}{l}\text { J. Activités } \\
\text { financières }\end{array}$ & & 0 & & 0 & 883638 & $6,08 \%$ & 4,50 \\
\hline $\begin{array}{l}\text { K. Immobilier et } \\
\text { SRE } \\
\text { (moins rente } \\
\text { imputée) }\end{array}$ & 5677 & 3,75 & 1301000 & 4,69 & 1042249 & $7,17 \%$ & 5,30 \\
\hline
\end{tabular}

Sources : SGJ 1980,1990, BHAS, BiH u brojevima 2010. 
Tableau 4. Emploi 1979, 1990, 2010

\begin{tabular}{|c|c|c|c|c|c|c|c|}
\hline & \multicolumn{2}{|c|}{$\begin{array}{c}\text { Emploi } \\
1979\end{array}$} & $\begin{array}{c}\text { Emploi } \\
1990\end{array}$ & \multicolumn{4}{|c|}{ Emploi 2010} \\
\hline & Abs & $\%$ & Abs & Abs & & $\begin{array}{l}\text { el'emploi } \\
\text { archand }\end{array}$ & $\%$ \\
\hline total emploi & 798000 & $100 \%$ & 1026254 & $100 \%$ & 684402 & & $100 \%$ \\
\hline total non marchand & 167400 & $20,98 \%$ & 193802 & $18,88 \%$ & 197975 & & $28,93 \%$ \\
\hline L. Administration & 35500 & $4,45 \%$ & 38391 & $3,74 \%$ & 69425 & & $10,14 \%$ \\
\hline M. Éducation & 58000 & $7,27 \%$ & 64044 & $6,24 \%$ & 56935 & & $8,32 \%$ \\
\hline N. Sanitaire et social & 38400 & $4,81 \%$ & 52976 & $5,16 \%$ & 45760 & & $6,69 \%$ \\
\hline O. Communal et social & 35500 & $4,45 \%$ & 38391 & $3,74 \%$ & 25855 & & $3,78 \%$ \\
\hline total marchand & 646400 & & 844127 & & 475364 & $100 \%$ & \\
\hline total prod matérielle & 439700 & $55,10 \%$ & 573502 & $55,88 \%$ & 232097 & $48,83 \%$ & $33,91 \%$ \\
\hline $\begin{array}{l}A+B \text {. Agriculture, forêts, } \\
\text { pêche }\end{array}$ & 36500 & $4,57 \%$ & 41032 & $4 \%$ & 18485 & $3,80 \%$ & $2,70 \%$ \\
\hline C. Mines & 33000 & $4,14 \%$ & 41272 & $4,02 \%$ & 19730 & $4,15 \%$ & $2,88 \%$ \\
\hline $\begin{array}{l}\text { D. Industries de } \\
\text { transformation }\end{array}$ & 265900 & $33,32 \%$ & 404437 & $39,41 \%$ & 133010 & $27,98 \%$ & $19,43 \%$ \\
\hline E. Énergie & 11900 & $1,49 \%$ & 11900 & $1,16 \%$ & 23310 & $4,90 \%$ & $3,41 \%$ \\
\hline F. BGC & 92400 & $11,58 \%$ & 74861 & $7,29 \%$ & 37562 & $7,90 \%$ & $5,49 \%$ \\
\hline total services marchands & 206700 & $25,90 \%$ & 270625 & $26,37 \%$ & 243267 & $51,17 \%$ & $35,54 \%$ \\
\hline $\begin{array}{l}\text { G. Commerce, services à la } \\
\text { personne }\end{array}$ & 99700 & $12,49 \%$ & 126384 & $12,32 \%$ & 127808 & $26,89 \%$ & $18,67 \%$ \\
\hline H. Hôtellerie & 28300 & $3,55 \%$ & 36757 & $3,58 \%$ & 29743 & $6,25 \%$ & $4,35 \%$ \\
\hline
\end{tabular}




\begin{tabular}{|l|c|c|c|c|c|c|c|}
\hline $\begin{array}{l}\text { I. Transports, } \\
\text { communications }\end{array}$ & 54400 & $6,82 \%$ & 68798 & $6,70 \%$ & 44027 & $9,30 \%$ & $6,43 \%$ \\
\hline J. Activités financières & 7800 & $0,98 \%$ & 11361 & $1,11 \%$ & 15418 & $3,24 \%$ & $2,25 \%$ \\
\hline $\begin{array}{l}\text { K. Immobilier et SRE } \\
\text { (moins rente imputée) }\end{array}$ & 16500 & $2,07 \%$ & 27325 & $2,66 \%$ & 26271 & $5,53 \%$ & $3,84 \%$ \\
\hline
\end{tabular}

Sources : SGJ 1980, 1990, BHAS 2011

Tableau 5. Emploi par branches $\mathrm{FBiH}$ et $\mathrm{RS}^{69}$

\begin{tabular}{|c|c|c|c|c|c|c|c|c|}
\hline & \multicolumn{3}{|c|}{ Chiffres absolus } & \multicolumn{5}{|c|}{ Chiffres relatifs } \\
\hline & FBH & RS & Total & $\begin{array}{c}\text { \%RS/ } \\
\text { BH }\end{array}$ & \multicolumn{2}{|c|}{$\begin{array}{c}\text { \% emploi en } \\
\text { FBH }\end{array}$} & \multicolumn{2}{|c|}{$\begin{array}{c}\text { \% emploi en } \\
\text { RS }\end{array}$} \\
\hline total salariés & \multicolumn{3}{|c|}{438949} & 244453 & 683402 & $35,77 \%$ & $100 \%$ & $100 \%$ \\
\hline total marchand & \multicolumn{3}{|c|}{304459} & 176821 & 481280 & $36,74 \%$ & $69,36 \%$ & $72,33 \%$ \\
\hline $\begin{array}{l}\text { A+B. Agriculture, forêts, } \\
\text { pêche }\end{array}$ & \multicolumn{3}{|c|}{9190} & 8632 & 17822 & $48,43 \%$ & $2,09 \%$ & $3,53 \%$ \\
\hline C. Mines & \multicolumn{3}{|c|}{14649} & 5022 & 19671 & $25,53 \%$ & $3,34 \%$ & $2,05 \%$ \\
\hline $\begin{array}{l}\text { D. Industries de } \\
\text { transformation }\end{array}$ & \multicolumn{3}{|c|}{84064} & 49014 & 133078 & $36,83 \%$ & $19,15 \%$ & $20,05 \%$ \\
\hline E. Energie & \multicolumn{3}{|c|}{13451} & 9515 & 22966 & $41,43 \%$ & $3,06 \%$ & $3,89 \%$ \\
\hline F. BGC & \multicolumn{3}{|c|}{26024} & 12774 & 38798 & $32,92 \%$ & $5,93 \%$ & $5,23 \%$ \\
\hline $\begin{array}{l}\text { G. Commerce, services à la } \\
\text { personne }\end{array}$ & \multicolumn{3}{|c|}{80969} & 46839 & 127808 & $36,65 \%$ & $18,45 \%$ & $19,16 \%$ \\
\hline H. Hôtellerie & \multicolumn{3}{|c|}{17084} & 11895 & 28979 & $41,05 \%$ & $3,89 \%$ & $4,87 \%$ \\
\hline $\begin{array}{l}\text { I. Transports, } \\
\text { communications }\end{array}$ & \multicolumn{3}{|c|}{29756} & 19983 & 49739 & $40,18 \%$ & $6,78 \%$ & $8,17 \%$ \\
\hline J. Activités financières & \multicolumn{3}{|c|}{11040} & 5108 & 16148 & $31,63 \%$ & $2,52 \%$ & $2,09 \%$ \\
\hline K. Foncier, leasing, SRE & \multicolumn{3}{|c|}{18232} & 8039 & 26271 & $30,60 \%$ & $4,15 \%$ & $3,29 \%$ \\
\hline & & & & & & & & \\
\hline
\end{tabular}




\begin{tabular}{|l|c|c|c|c|c|c|}
\hline total non marchand & 130631 & 67344 & 197975 & $34,02 \%$ & $29,76 \%$ & $27,55 \%$ \\
\hline L. Administration & 46873 & 22552 & 69425 & $32,48 \%$ & $10,68 \%$ & $9,23 \%$ \\
\hline M. Education & 37324 & 19611 & 56935 & $34,44 \%$ & $8,50 \%$ & $8,02 \%$ \\
\hline N. Sanitaire et social & 29235 & 16525 & 45760 & $36,11 \%$ & $6,66 \%$ & $6,76 \%$ \\
\hline O. Communal et social & 17199 & 8656 & 25855 & $33,48 \%$ & $3,92 \%$ & $3,54 \%$ \\
\hline
\end{tabular}

Sources : T6.2 in SG FBiH 2010, T6.6 in SG RS $2010^{70}$ (rappel : population RS/BH = 37,4\% - VA RS/BH $=33,79 \%)$

\section{BOX : Mode de reclassement des rubriques}

La classification internationale regroupe désormais A « Agriculture et sylviculture », la pisciculture étant traitée à part (rubrique $\mathrm{B}$ ).

La rubrique «Industrie et mines» se trouve séparée dans trois rubriques : C, « Mines », D, «Industries de transformation », $\mathrm{E}$, «Énergie et Réseaux ». Dans notre cas, ceci amène à incorporer dans la rubrique $\mathrm{E}$ l'ancienne rubrique « Hydraulique».

La rubrique $\mathrm{G}$ «Commerce, réparations automobiles, services aux ménages », incorpore dorénavant la catégorie "sociale" « Artisanat " (zanatstvo, obrtništvo) ${ }^{71}$.

La rubrique $\mathrm{H}$ « Hôtellerie » exclut dorénavant les services touristiques, renvoyés dans la rubrique $\mathrm{K}$.

Il en va de même pour la rubrique I « Transports et communications », qui renvoie désormais les services de stockage en $\mathrm{K}$.

La rubrique J «Finances», reprend «Finances et Assurances». Ces dernières - par excellence improductives dans une logique marxiste - correspondent à une case vide dans la situation actuelle.

La rubrique K, nouvelle, dite «Services immobiliers, leasing et autres services aux entreprises ", regroupe en fait des activités diverses. D'une part elle reprend les activités immobilières, locations (donc le logement social, stambena komunalna djelatnost), les géomètres et autres agents immobiliers. Mais elle comprend également le leasing, et surtout, tous les services rendus principalement aux entreprises, les SRE dans notre terminologie, donc l'informatique, la communication etc. L'étude de cette rubrique fait apparaître une inclusion purement statistique pour l'établissement des comptes nationaux: l'apparition d'une rente foncière imputée, pour égaliser le coût du logement entre propriétaires et locataires. Mais dans un pays où 91\% de la population est propriétaire, le décalage est important entre la dimension statistique et celle des transactions monétaires.

La rubrique L « Administrations » permet de récupérer tout ce qui était DPO (društvene političke organizacije), c'est-à-dire l'administration propre du temps de l'autogestion (ce qui a sans doute impliqué une réallocation des SIZ - samoupravne interesne zajednice - communautés d'intérêt autogestionnaires, qui géraient les services collectifs, entre leurs divers domaines d'action). Elle inclut également les activités communales courantes (voirie, services de secours).

$M$ et N, respectivement " Éducation » et « Santé », ne posent pas de problèmes particuliers.

Note: la rubrique K. originelle comporte la rente imputée pour les propriétaires occupants, afin de préserver l'homogénéité des données et d'éliminer le principal facteur d'économie non observée. Mais cette manière de procéder biaise les comparaisons temporelles, ainsi que l'évaluation de la richesse circulant réellement ; pour ces raisons la rente imputée a été éliminée. 

abandonné un modèle bureaucratique, l'administration publique a considérablement cru (68 425 personnes, contre 38391 en 1990). Il est vrai que la Yougoslavie autogestionnaire avait l'art de cacher l'administration dans les entreprises, sous couvert d'autogestion. Les délégués dans les divers conseils autogestionnaires ainsi que les multiples SIZ, la Défense populaire (NO), sans parler de la Ligue, des syndicats et du SSRN n'étaient pas surchargés de tâches de production. Néanmoins, on peut s'étonner qu'avec une chute de près d'un quart de la population et une natalité en berne, le nombre des administratifs ait augmenté de $80 \%$ depuis 1990, alors que le régime socialiste avait réussi, au moins formellement, à en contenir l'expansion pendant la crise économique 1980-1990. L'édifice institutionnel de Dayton semble avoir un coût administratif ${ }^{82}$ tout à fait appréciable. On remarquera par ailleurs que l'organisation unitaire de la RS semble moins budgétivore que celle de la Fédération $(9,23 \%$ des emplois contre 10,67\%), sans doute en raison, pour la $\mathrm{FBiH}$, de la charge des cantons et de l'administration générale du pays, concentrée à Sarajevo.

Il faut maintenant envisager les différences entre les entités. On constate un étrange parallélisme de comportement entre les entités. La distinction principale tient à l'agriculture, qui conserve une grande importance en RS : (10,7\% du PIB de la RS, contre $5,1 \%$ en Fédération $\left.{ }^{83}\right)$. La différence est même plus frappante, si l'on tient compte de l'emploi de la population : si la population agricole se monte à $19,7 \%$ dans le pays en 2010 , elle atteint $31,5 \%$ en RS, contre $12,2 \%$ en FBiH. Dans les deux cas, on remarque le caractère de stockage de population par rapport à la production, surtout si 
l'on tient compte de la plus grande possibilité d'agriculture intensive en RS dans sa partie de plaine.

Dans les autres domaines de production, des variations existent, mais elles ne mettent pas en évidence des différences structurelles décisives, si ce n'est que les mines ont moins d'importance en RS, mais que le secteur énergétique y prend approximativement la même part qu'en Fédération. Ni la démographie, ni l'emploi, ne connaissent de dynamiques sensiblement différentes. D'ailleurs les parts de production des entités restent assez stables au cours du temps et, on le verra, leurs modes de régulation économique présentent des analogies étonnantes, à quelques variations près (ampleur des privatisations et par conséquent des IDE plus importante en RS).

En tout cas, aucune des entités ne s'est engagée dans un sentier de développement rompant avec les pratiques de l'ère socialiste. Ce non-choix, que l'on retrouve dans la pratique des $\mathrm{IDE}^{84}$, mais aussi dans les politiques éducatives et scientifiques, frappe par sa contradiction avec les prétentions politiques des deux entités. Le développement de la $\mathrm{BiH}$ actuelle dans ses deux composantes ne repose donc ni sur une agriculture productive, ni sur l'économie du savoir, au sens du Programme de Lisbonne ${ }^{85}$. L'ensemble du système semble reposer dans le domaine industriel sur une survie quelque peu automatique à partir du patrimoine naturel et industriel existant, avec des succès tenant à la capacité des managements d'entreprise à se réinsérer dans les flux régionaux et européens ${ }^{86}$.

Dans le domaine de l'immatériel, aucune activité tertiaire motrice ne semble avoir pris le relais, ce qui se répercute dans les faibles effectifs des activités correspondantes, sans changement notable par rapport à l'époque où ces activités étaient intégrées au sein des groupes. L'exception notable reste la firme d'ingénierie Energoinvest, au périmètre bien réduit.

TABLEAU 6. PIB RELATIF DES ENTITÉS 2005-2010 EN \%

\begin{tabular}{|l|c|c|c|c|c|c|c|}
\hline & $\mathbf{2 0 0 5}$ & $\mathbf{2 0 0 6}$ & $\mathbf{2 0 0 7}$ & $\mathbf{2 0 0 8}$ & \multicolumn{2}{|l|}{$\mathbf{2 0 0 9}$} & \multicolumn{2}{|l|}{$\mathbf{2 0 1 0}$} \\
\hline & & & & & & \multicolumn{1}{|l|}{} \\
\hline PIB BiH & 100 & 100 & 100 & 100 & 100 & 100 \\
\hline & & & & & & & \\
\hline PIB FBiH & 63,92 & 63,74 & 63,83 & 63,36 & 63,52 & 63,91 \\
\hline PIB RS & 33,47 & 33,85 & 33,66 & 34,29 & 34,19 & 33,79 \\
\hline PIB Brčko & 2,61 & 2,42 & 2,51 & 2,35 & 2,29 & 2,29 \\
\hline
\end{tabular}

Source : BHAS Tematski Bilten TB 01-2012, BDP po rashodnom pristupu 2005-2010

L'ensemble du système productif montre un blocage, tant quantitatif que qualitatif. Quantitativement, les investissements sont à peine au niveau des amortissements (en 2010, 4 221,965 millions de KM d'investissements, 3 557,559 millions de KM d'amortissements ${ }^{88}$ ). Les entreprises, comme les charbonnages (rattachés à l'entreprise d'électricité), ne parviennent pas à effectuer une simple mise à jour de leurs 
équipements pour réaliser des gains de productivité et de sécurité. Quant à l'ancien secteur de pointe, celui des équipements militaires, il se trouve en situation de blocage. Ces données se retrouvent dans les comptes nationaux au niveau de l'EBE (excédent brut d'exploitation, bruto poslovni višak), qui fait apparaître le secteur minier en pertes, et le secteur industriel ne fournissant que 9,6\% des excédents d'exploitation de l'ensemble de l'appareil économique. Le système industriel est donc semble-t-il en survie : stockant de l'emploi dans des conditions de rémunération insatisfaisantes, il ne parvient à se renouveler ni en fonction des marchés, ni en fonction des technologies.

La question du renouvellement productif est largement liée aux IDE, fournisseuses de compétences et de technologies. Ces dernières ont eu une contribution plus que modeste à la croissance du pays depuis $1996^{89}$. En effet, sans insister sur le retard des privatisations qui restent à effectuer, il faut rappeler que beaucoup d'entreprises bosniaques ont un capital fermé, avec une préférence nette pour le statut de Sarl (d.o.o, društvo sa ograničenom orgovornošću), et cherchent à réaliser leurs projets au travers de joint-ventures. Les forums d'investisseurs mentionnent systématiquement des " projets " proposés à la participation, sans qu'on n'évoque jamais la solution habituelle dans le monde occidental de la prise de participation, éventuellement croisée. Or, les investisseurs étrangers ont des stratégies de firmes, et cherchent à maîtriser la gestion des projets auxquels ils participent. Ils se tiennent donc en retrait par rapport aux propositions. La moitié des IDE ont eu pour cible le secteur financier désormais largement intégré aux réseaux européens, ce qui a profité à l'économie dans son ensemble, et qui a limité les dérives financières. Dans l'industrie, les grands moments ont été la reprise des aciéries de Zenica (et des mines de fer de Vareš et Ljubija, le seul projet parfaitement inter-entités ${ }^{90}$ ), la privatisation des télécommunications de RS par Télécom Serbie, celle de la raffinerie de B. Brod par le groupe russe Zarubezhneft (avec reprise du réseau de distribution Zovko), la fabrique de carton de Maglaj par le groupe turc Hayat.

Les industries qui ont pu retrouver des conditions normales d'activité sont presque toutes liées à des biens intermédiaires, avec intervention de capitaux étrangers. Rares sont les exceptions, liées à des initiatives des directions (Borač Travnik, Prevent Visoko et VW à Goražde), et nombre de firmes restent dans des situations instables avec capitaux publics (Birac, Aluminij, VTK, Krivaj, pour celles qui sont le plus fréquemment mentionnées). Le BTP souffre à l'évidence de l'absence de relais de demande postérieurement à la reconstruction: la démographie atone limite la construction de logements, le caractère chaotique des grands investissements (corridor Vc), le manque d'investissements industriels, n'assurent pas un volume d'affaires suffisant pour rétablir le marché ( $19 \%$ de l'activité du BTP dans le logement, pour 3278 logements achevés en $2009^{91}$ ).

On pourrait faire observer que les tendances historiques, amorcées sous les Habsbourg, en partie poursuivies avec le socialisme, se sont en définitive perpétuées : exportation sur base de ressources naturelles, émigration, agriculture de montagne structurellement faible. La déstabilisation quantitative de l'économie due à la guerre a été amortie. La déstabilisation qualitative est plus importante, et on peut la lier à l'extinction de la Yougoslavie comme puissance d'influence dans le contexte international. Les firmes disparues ou radicalement réduites correspondent soit aux marchés régionalisés de l'autogestion fédéralisée, soit à l'armement (UNIS, Soko), soit à la fin des non-alignés, et malgré toute l'attention portée au pays par de nombreuses 
institutions et pays amis, la configuration des marchés n'a pas jusqu'à présent été favorable au renouvellement du tissu industriel bosniaque.

Les experts internationaux font fréquemment figurer l'environnement institutionnel défavorable comme cause des blocages de développement, mais ceci ne concerne que les stratégies des investisseurs extérieurs (IDE, et entreprises foot loose, sans enracinement particulier), ou le développement local des petites et moyennes entreprises (SME). La question est plutôt donc celle des dynamiques internes au pays et à ses entités, et plus particulièrement celle de l'attitude du corps social vis-à-vis du renouvellement du tissu industriel et de services.

Or, l'emploi se ressent de cette situation d'immobilisme, même en tenant compte de l'emploi informel et du départ en retraite des premières générations d'emploi de masse. Si le niveau de production est revenu à 1990, celui de l'emploi salarié est, avec 816000 postes, revenu à 1980 (820 000), celui de la population apte au travail à 1976-1977, avec 2561000 personnes $^{92}$. On peut donc légitimement s'interroger sur les moyens d'existence que les structures économiques assurent à la population.

1. 1.

\subsection{Un niveau de vie qui a progressé, sur quelles ressources?}

Contrairement à une vision pessimiste de la société que suscite l'analyse des structures productives, on constate que le niveau de vie a nettement augmenté depuis la fin de la Yougoslavie. L'alimentation est à un niveau satisfaisant $(3084 \mathrm{cal} / \mathrm{j}$, avec des rations de protéines animales suffisantes dues aux produits laitiers $\left.{ }^{93}\right)$, moins de $5 \%$ de population est sous-alimentée. L'espérance de vie s'élève à 75 ans, et la mortalité infantile est de 14 p.m. améliorée par rapport à celle de 1990 (15,2 p.m.) ; ces données sont cohérentes avec celles de l'alimentation. Le taux de pauvreté n'est pas critique, se situant à $15 \%$ de la population, avec absence statistique de situations de pauvreté absolue ${ }^{94}$.

Sur le plan matériel, le progrès est net. En premier lieu, témoignage de développement, la part du budget des ménages consacrée à l'alimentation est passée de 46,2\% en 1989 à $32 \%$ en 2010. Et dans ce budget alimentaire, la part des céréales s'est réduite (17\%), le poste céréales n'étant plus que le $7^{\text {ème }}$ dans la valeur des importations alimentaires du pays. Au niveau des biens matériels et du confort de vie, la surface habitable est passée de $15,3 \mathrm{~m}^{2} / \mathrm{h}$ en 1981 à $28,1 \mathrm{~m}^{2} / \mathrm{h}$, pour $91 \%$ de propriétaires, et un taux de raccordement de plus de $99 \%$ au réseau électrique. La motorisation s'est étendue (augmentation de 65\% du nombre de véhicules particuliers, un parc de 725000 véhicules, soit près des trois-quarts des ménages), et l'équipement ménager s'est généralisé (taux d'équipement passé de $88,6 \%$ à $97,6 \%$ en réfrigérateurs, de $57,2 \%$ à $86,1 \%$ en machines à laver, de $84 \%$ à $96,8 \%$ en téléviseurs).

On ne peut négliger la part qu'ont pu prendre la politique de change (rivage du KM à un euro fort) et l'ouverture du commerce extérieur (ASA avec l'UE, CEFTA) dans cette amélioration des conditions de vie, en facilitant l'accès aux biens importés, et en garantissant la valeur des revenus. L'accès au crédit, facilité par l'arrivée de banques européennes, a également eu sa part dans l'évolution. Ces données pourraient évoquer une croissance tirée par la consommation, ce qui a certainement été le cas au début des années 2000, mais il faut alors prendre en considération les racines de cette relative aisance, et rapporter celle-ci à l'activité économique. 
71 Nous avons tenté de reconstituer, sans prétention à un véritable bouclage comptable, les ressources disponibles pour la consommation en 2010, en commençant par les salaires.

Le compte de consommation de la comptabilité nationale (GDP po rashodnom pristupu, PIB à partir de la consommation ${ }^{95}$ ) nous indique une consommation des ménages de 21828 millions de KM. Or, la BiH comptait 816000 salariés en $2010^{96}$, qui gagnaient 800 KM par mois en moyenne, ce qui induit un montant solvable de 7 833,6 millions de KM. Or, le panier de la ménagère se monte à environ $1835 \mathrm{KM} / \mathrm{mois}^{97}$, ce qui voudrait dire que, sauf à disposer de deux salaires par famille, il serait impossible de satisfaire la consommation de base. Pour les nombreux retraités (572 000 en 2010), la mensualité moyenne est de $350 \mathrm{KM}$, ce qui correspond à une somme globale de 2402 millions de KM.

Le décalage étant très important entre les salaires et la consommation, nous avons tenté de reconstituer les sources de revenus qui assurent la solvabilité.

74 Les salaires et retraites ne sont pas les seuls contributeurs au revenu des particuliers. L'aide sociale, dont près des deux tiers (62,6\%) vont aux pensions d'anciens combattants, absorbe - en tenant compte des interventions des collectivités locales $8 \%$ du PIB, ce qui donnerait en 2010, 1924 millions de $\mathrm{KM}^{98}$.

Il n'en reste pas moins un différentiel important, qui comprend en premier lieu une part correspondant aux profits du secteur privé, dans lequel nous englobons l'agriculture, une grande partie du commerce et des services à la personne, des transports, de l'hôtellerie, des services, et qui reprend de manière comptable la consommation de logement des propriétaires occupants. En calculant à partir de la colonne profits nets du compte de production (tableau 5.5 des comptes nationaux 2005-2010, mais à vrai dire sans déduire les profits des entreprises d'Etat, Télécoms et Chemins de fer essentiellement ${ }^{99}$ ), nous trouvons 4204 millions de $\mathrm{KM}^{100}$.

On peut faire rentrer en jeu le travail non observé (presqu'entièrement constitué de sommes disponibles pour la consommation, évalué par la Banque centrale en 2008 à environ $20 \%$ du $\mathrm{PIB}^{101}$ ) et l'on aboutit selon les calculs de Rajko Tomaš ${ }^{102}$ à 2600 millions de KM.

77 C'est à ce stade qu'interviennent les revenus de l'étranger. En effet, ces derniers, chiffrés à 3200 millions de KM, dont 2100 millions de KM de remises de travailleurs (le reste étant, selon la Banque centrale, largement composé de retraites versées à des résidents $\left.{ }^{103}\right)$. Ces remises sont d'autant plus essentielles qu'elles financent des dépenses non quotidiennes ${ }^{104}$, comme la santé et l'éducation. La part mentionnée pour les investissements (un tiers des remises) semble avoir un sens avant tout statistique, car il pourrait bien s'agir de l'investissement des ménages (FBCF), c'est-à-dire de la construction de logements.

Tableau 7. Sources de revenus en $\mathrm{BiH}$

\begin{tabular}{|l|c|}
\hline Sources de revenu & $\begin{array}{c}\text { Montant } \\
\text { (millions KM) }\end{array}$ \\
\hline & \\
\hline TOTAL & 21063 \\
\hline
\end{tabular}




\begin{tabular}{|l|c|}
\hline & \\
\hline Salaires nets & 7833 \\
\hline Retraites & 2402 \\
\hline Transferts sociaux & 1924 \\
\hline Remises étranger & 2100 \\
\hline Profits individuels & 4204 \\
\hline Activité grise & 2600 \\
\hline
\end{tabular}

78 On retombe donc approximativement sur le niveau de consommation constaté. Au total, les revenus distribués par les entreprises semblent avoir conservé une place modérée dans les capacités de consommation, par rapport à d'autres ressources formelles, toutes contrôlées par l'État.

La vision de la $\mathrm{BiH}$ comme une société salariale reposant sur son industrie se trouve donc passablement effritée. Par contre, on constate très largement une société de redistribution: sur 12200 millions de KM issus des trois postes salaires, pensions et transferts sociaux, réaffectant la valeur produite par les entreprises, $35 \%$ sont redistribués. L'activité privée ne représente qu'environ $20 \%$ du revenu, en y comptant la paysannerie. Mais si l'on fait la somme des trois postes « individuels » qui échappent à l'État, à savoir les remises de l'étranger, les profits et l'économie grise, on trouve $42 \%$ des revenus disponibles.

On se trouve donc dans une situation où, par rapport au salariat, l'État redistribue des sommes importantes à la population inactive (sauf aux observations concernant les pensions de guerre ${ }^{105}$ ), et où les revenus salariaux constituent une part importante, mais pas hégémonique, de l'ensemble des sommes disponibles.

Dans cet ensemble, l'État semble à première vue détenir une place modeste : l'essentiel des retraites et de la santé est financé sur cotisations, et les aides sociales sont plutôt modestes en volume, même si leur coût budgétaire semble hors de portée des ressources de l'État. Mais nous allons voir que le pouvoir de la puissance publique sur les revenus dépasse largement ce cadre.

1.

\section{La place de l'État}

1.

\subsection{L'État au contrôle de la formation et de la redistribution des revenus}

Il n'y a plus de direction de l'économie par l'État, ni au niveau central, ni au niveau des entités ou des cantons. Cependant, l'État s'est maintenu dans l'économie productive au travers de ses participations, certaines résiduelles (pas d'acheteurs), certaines stratégiques ou considérées comme telles (essentiellement des branches de base, ou du secteur des infrastructures). Comme nous l'avons observé plus haut, ces participations ne génèrent pas de profits élevés. Elles sont plutôt motivées par les «intérêts 
supérieurs de la nation ", avec les modulations habituelles en BiH sur ce terme selon les entités et les groupes de référence.

C'est pourquoi, dans le cas bosniaque, où les rentes naturelles existent certes, mais n'atteignent pas le niveau nécessaire au développement d'un pays ne serait-ce que de moins de 4 millions d'habitants, on ne peut expliquer la place de l'État dans l'appareil productif par un souci d'assurer l'avenir.

Par contre, l'État s'est glissé au cœur du système de redistribution. Les participations d'État n'assurent pas de revenus, ne préparent pas l'avenir, mais elles induisent le pouvoir de direction sur une main-d'œuvre nombreuse.

Ces emplois viennent se cumuler avec ceux que contrôle, dans un rôle a priori classique, l'État employeur de ses fonctionnaires. Mais ce cumul est démultiplié par l'existence d'une fonction publique surnuméraire qui vient s'ajouter à des entreprises publiques elles-mêmes en situation de suremploi. Le total est éloquent: la fonction publique élargie (secteurs L, M, N, O) représente $28,93 \%$ de l'emploi, à quoi viennent s'ajouter au moins les mines et l'énergie, soit $6,29 \%$, et une bonne partie de l'industrie de transformation, dans l'aluminium, les industries de défense, l'ingénierie électrique par exemple; vient encore s'y ajouter les chemins de fer, les autoroutes et l'entretien routier, ainsi que les télécommunications (8 500 personnes dans la seule $\mathrm{FBiH}$ ). C'est donc certainement au-delà du tiers, et sans doute près de $40 \%$ de l'emploi qui se trouve directement entre les mains de l'État, État entitaire certes, mais État tout de même.

Dans les entreprises privées à participation minoritaire, le pouvoir de direction est délégué au management, sous réserve d'une sauvegarde de l'entreprise, mais dès qu'une situation conflictuelle avec le personnel apparait, les syndicats rentrent en $\mathrm{jeu}^{106}$, selon une tradition déjà bien établie à l'époque du pouvoir autogestionnaire. C'est alors à l'État que revient la négociation, qui peut aboutir, comme dans les charbonnages, à des réorganisations et à la prise en charge des dettes publiques, ou à des négociations tarifaires avec l'entreprise d'électricité (EP/aluminium) ou les chemins de fer (Željeznice RS). Il est cependant à remarquer que la faible assiette budgétaire disponible ne permet guère de poursuivre la tradition socialiste de la socialisation pure et simple des pertes ${ }^{107}$. Les négociations se déroulent donc dans une espèce de louvoiement entre niveau d'emploi, rémunérations, et gestion du passif financier.

8 En définitive, c'est une grande partie de la masse des salaires qui se trouve régulée par les entités et souvent par les cantons, les actions syndicales ayant souvent pour objet les annexes du salaire (indemnités diverses, avantages en nature). Dans le domaine propre de l'État, il est unanimement estimé que les dépenses de fonction publique sont élevées, (12,9 \% du PIB, à comparer aux 15,4 \% consacrés par cet appareil aux transferts sociaux $)^{108}$, tant en raison des effectifs que des grilles de rémunérations.

Avec des prélèvements obligatoires au niveau de la moitié du PIB ${ }^{109}$, et ce malgré la modestie des taxes sur les salaires et les profits (au taux de $10 \%$ ), $85 \%$ de ces derniers sont canalisés vers les dépenses courantes. Étant donné qu'outre l'administration courante, l'essentiel de l'action publique consiste à répartir des transferts en direction de publics réputés fragiles (dont nous avons estimé le niveau plus haut), l'État se donne des moyens d'agir également sur des populations se situant hors du champ salarial. Par la part prépondérante de la cible des anciens combattants, le pouvoir assure un lien plus politique qu'économique ou social. Ce lien s'étend aux agriculteurs, les revenus 
agricoles étant largement structurés par diverses subventions, dont celles qui visent le secteur clé du lait ${ }^{110}$. une caractérisation économique possible serait celle d'économie résidentielle - ou plutôt, comme l'exprime L. Davezies ${ }^{113}$, celle d'économie présentielle, particulièrement applicable aux régions d'implantation de retraités, mais également aux pays de grande émigration. Certes, le facteur extérieur est déterminant dans les possibilités de consommation en $\mathrm{BiH}$, mais on ne peut pas considérer que l'appareil économique est la résultante de services aux résidents tirant leurs revenus d'autres territoires, et ce malgré l'importance du nombre de retraités. corporatif ${ }^{114}$ qui assure une certaine stabilité à ce qui reste du système industriel ${ }^{115}$, sans grande possibilité d'influence des actionnaires, souvent eux-mêmes salariés des mêmes entreprises. Une série de compensations internes, proches des ajustements de l'ère socialiste, assurent une continuité de survie, en effectuant des prélèvements sur une consommation relativement ample. Mais le monde industriel ne bénéficie plus du privilège d'être "prolétarien ", et les compensations se masquent derrière la solidarité sociale. L'essentiel de ces compensations est financé par l'extérieur du monde industriel. Comme dans l'ancienne Yougoslavie, la viabilité d'une économie déficitaire est assurée par des ressorts externes. Remises de l'étranger, paysannerie en autosuffisance, exportations de biens bruts ou de première transformation, marginalisation de l'initiative économique dans l'économie parallèle.

Mais en conséquence de ce choix de "glaciation", les freins sont nombreux pour un développement de long terme: niveau plancher pour les investissements nationaux, quasi fermeture aux investissements étrangers, insuffisance du réseau national d'innovation, obstacles à la création d'entreprises concurrentielles d'une certaine ampleur. 

économique, ce dont ont témoigné les mouvements agrariens qui ont précédé le communisme. En ce qui concerne le rapport du pouvoir à la redistribution, il conviendrait pour la $\mathrm{FBiH}$ de mentionner la tradition soufie, qui a longtemps irrigué la 
Bosnie et l'Herzégovine, avec maintenant une réappropriation par le politique du rôle du cheikh, détenteur d'autorité mais en même temps dispensateur de gratifications. A. Henni a étudié ce rôle chez les directeurs d'entreprises algériennes ${ }^{121}$ et Youssef Belal y a trouvé les racines du renouvellement du pouvoir au Maroc ${ }^{122}$. Mais pour la RS, l'explication tombe court, et pourtant on se trouve face au même comportement. On peut certes faire référence au passé ottoman commun, mais on pourrait évoquer sous toutes réserves la tradition de discipline de la Marche militaire, qui contraste avec l'individualisme paysan serbe de Serbie (Srbijanska).

L'équilibre économique instable de la $\mathrm{BiH}$ permet provisoirement aux aspirations nationales de se satisfaire de la politique parallèle menée par les institutions entitaires. Cet équilibre se nourrit de l'absence de pression démographique, et de l'existence d'une vaste diaspora encore attachée à son pays de référence. Mais face à cet équilibre fragile, la politique de normalisation des institutions menée par les instances internationales n'est pas assurée de voir se développer un nouvel entrepreneuriat orienté vers la compétitivité et l'économie de la connaissance. Trop d'intérêts corporatifs associés à un idéal rentier peuvent continuer à faire croire que le système redistributif actuel est, sinon le meilleur, tout au moins celui qui protège le mieux les aspirations nationales. La rupture viendra peut-être lorsque le manque de ressource humaine et l'éloignement progressif de la diaspora mettront le pays face à d'autres enjeux démographiques.

1.

\section{Conclusion}

La Bosnie-Herzégovine, république fédérée au sein de la Yougoslavie socialiste fédérale, présentait un visage ambivalent, entre succès d'une modernisation sociale accompagnée d'une lente stabilisation démographique, et relatif échec d'une croissance économique assise sur sa situation géostratégique et ses ressources naturelles. Cette croissance ne permettait pas, hors processus d'émigration facilité par l'intégration culturelle, de faire face aux excédents démographiques résultant des périodes précédant la transition démographique.

Le conflit 1992-1995 a supprimé la partie spécifiquement fédérale (le militaire) du complexe construit dans l'après-guerre, mais il a également entraîné une rupture démographique, tant par l'émigration forcée que par le blocage de l'expansion démographique. De ce fait, l'appareil de production matérielle a certes prolongé son existence, mais sans offrir de perspectives à long terme ; la tertiarisation de l'économie s'est basée sur une libéralisation de l'activité de services, elle-même bornée par une solvabilité dépendant largement des remises de l'étranger, sans génération de services exportateurs à destination des entreprises.

Cette orientation semble affecter de manière identique les deux entités, qui ne semblent en aucun cas en concurrence pour un nouveau mode de développement. L'une comme l'autre ont organisé un système redistributif qui permet de limiter la pauvreté extrême, et qui surtout conforte l'affirmation identitaire.

Ceci expliquerait alors la référence insistante aux ressources de la nature, cette dernière justifiant mythiquement un système de distribution inspiré des États rentiers, en général dotés d'une rente en hydrocarbures. Il faudra aller plus loin dans l'analyse des discours pour saisir comment les deux entités cherchent leurs références, l'une chez la Russie, l'autre chez les États centre-asiatiques ou moyen-orientaux avec lesquels ils se sentent des affinités culturelles ${ }^{123}$. 


\section{NOTES}

1. Ganev (Venelin I.), Preying on the State. The transformation of Bulgaria after 1989, Ithaca : Cornell University Press, 2009 ; Bojičić-Dželilović (Vesna), Kostovica (Denisa), Persistent State Weakness in a Global Age, London : Ashgate, 2009.

2. Nous laisserons de côté l'autonomisme croato-herzégovinien, qui ne semble pas présenter de projet économique structuré.

3. Boltanski (Luc), Thévenot (Laurent), De la justification, les économies de la grandeur, Paris: Gallimard, 1991.

4. Kornai (Janos), L'Économie de la pénurie, Paris : Economica, 1982 ; Uvalić (Milica), Investments and Property Rights in Yugoslavia. The Long Road to a Market Economy, Cambridge: Cambridge University Press, 1992.

5. Glamočak (Marina), La transition guerrière, Paris : La Découverte, 2001.

6. On n'a pas assez souligné qu'à part en Slovénie, il n'y a pas eu de "Révolution de velours ", surtout pas en BiH. Le poids des réformes compromissoires d'A. Marković, et donc de l'ancien système des SOUR, nous semble encore marquant dans les structures d'entreprise du pays.

7. Nous ne nous risquerons pas à trancher sur le terme le plus approprié, le champ de ce travail ne comportant pas la nécessité d'hypothèses sur ce sujet.

8. Istraživački Dokumentacioni Centar/Norvegian Ministry of Foreign Affairs, Ljudski gubici u Bosni i Herzegovini 91-95, Sarajevo, 2009.

9. Sur la question du retard du à la guerre, voir IMF (2005).

10. Dans le cadre de ce travail, la dénomination d'usage du temps de la Yougoslavie nous semble plus pratique que la dénomination diplomatique d'ARYM/FYROM. Elle reste d'usage courant à l'intérieur des frontières de l'ex-Yougoslavie.

11. Selon Savezni Zavod za Statistiku SFRJ, Statistički Godišnjak SFRJ, 1990, p. 411.

12. La question linguistique de la minorité albanaise ne se retrouve pas en $\mathrm{BiH}$, et la question religieuse transcende celle des langues. Enfin, l'identité macédonienne se cimente contre les identifications extérieures, à l'inverse de l'assimilationisme serbe ou croate en BiH.

13. Selon la CNUCED, PIB 2010 : BiH 4478 \$/h, ARYM 4434 \$/h, Serbie 4394 \$/h, Monténégro 6510 \$/h (unctadstat.unctad.org/table viewer/).

14. Pettifer (James), « The news Macedonian question », International Affairs, 68 (3), 1992.

15. Bien qu'un effritement se soit dessiné dans tout l'après-guerre mondiale, on le verra plus loin.

16. Sachter (Hugues), Développement et aménagement de territoire dans la Yougoslavie socialiste, Paris: Université Paris I (Thèse pour le doctorat de Sciences Économiques), miméo, 1982 ; Sachter (Hugues), "Quelques aspects économiques de la dimension régionale de la crise en Yougoslavie ", in Lavigne (Marie), Andreff (Wladimir), La Réalité Socialiste, Paris : Economica, 1985. 17. EBRD, Transition Report. Country Assessments: Bosnia and Herzegovina, 2009.

18. Lydall (Harold), Yugoslavia in Crisis, Oxford : Oxford University Press, 1989.

19. Savezni Zavod za Statistiku SFRJ, op.cit.; Savezni Zavod za Statistiku SFRJ, Statistički Godišnjak SFRJ, 1980.

20. Kubović (Branko), Ekonomska Historija Yugoslavije, Zagreb: Informator, 1971. Même la raffinerie de $\mathrm{B}$. Brod date de ce temps, sans doute dans une conjoncture géographique liée à la concurrence réciproque austro-hongroise.

21. Le rapport de la Banque mondiale de 1975 (rapport Schrenk), intervient au moment où ce type de financements devient quasiment impossible. C'est d'ailleurs au $\mathrm{X}^{\circ}$ congrès de la LCY que fait paradoxalement surface le thème plutôt « maoïste »de « compter sur ses propres forces ». 
22. Woodward (Susan L.), Socialist Unemployment. The Political Economy of Yugoslavia 1945-1990, Princeton : Princeton University Press, 1995

23. OCDE, Country Study Yugoslavia 1986, Paris : OCDE, 1987.

24. SKJ, XI. kongres Saveza komunista Jugoslavije, Beograd : Komunist, 1974.

25. Sauf aux confins nord (Semberija) et sud (Neretva).

26. Milivojević (Marko), Yugoslavia's Military Industries, Bradford : University of Bradford, 1990 ; JNA, « JNA u izgradnji zemlje 1960-1987 », Jugoslovenski Pregled, 11-12, 1987, pp. 490-491.

27. Cf. pour le complexe métallurgique serbe : Palairet (Michael), «The Metallurgic Combinate Smederevo 1960-1990. A Case Study in the Decline of Yugoslavia », Europe-Asia studies, 47, 1997.

28. Stevanović (Radoslav), Breznik (Dušan), "The population of Yugoslavia, 1948-1991", Yugoslav Survey, 32 (3), 1991.

29. Savezni Zavod za Statistiku SFRJ, op.cit., 1990.

30. Woodward (Susan L.), op.cit.

31. Toujours la peur des koulaks, renforcée par la rémanence des thèses agrariennes (narodnjaci) toujours marquées au coin du communautarisme nationaliste.

32. Palairet (Michael), The Mismanagement of the Yugoslav Rural Economy, 1945-1990, unpublished manuscript, 1996 (sur esiweb.org/pdf/bridges/bosnia, consulté le 22/02/2012)

33. Savezni Zavod za Statistiku SFRJ, op.cit., 1990, T 203.-18.

34. Stevanović (Radoslav), Breznik (Dušan), art.cit., TA4 ; mais le tableau Savezni Zavod za Statistiku SFRJ, op.cit., 1990, T 203-18 permet de calculer près de 350000 personnes de 1945 à 1981

35. Savezni Zavod za Statistiku SFRJ, Statistički Godišnjak SFRJ, 1991, T 203-1.

36. L'évaluation est délicate en ce qui concerne la Slovénie, qui n'avait reçu (mêmes sources SGJ 1990) que 30000 personnes de B\&H en 1981, alors que, selon nos entretiens de l'époque, un nombre bien supérieur de gens travaillaient en Slovénie, mais continuaient à être comptés comme résidents bosniaques pour des raisons de prestations sociales aux familles restées au pays. Le chiffre actuel de 100000 personnes d'origine bosniaque résidant en Slovénie (Ministry of Security of Bosnia and Herzegovina, Migration profile 2009, 2009) incorpore sans doute nombre de gens stabilisés, souvent avec leurs familles, désormais comptés comme réfugiés.

37. Stevanović (Radoslav), Breznik (Dušan), art.cit., T A-11.

Et encore ce chiffre peut-il contenir des Sandžaklije de Serbie et du Monténégro, réputés mobiles, et des Musulmans du Kosovo et de Macédoine.

38. Čolanović (Branko), Le développement des régions insuffisamment développées en Yougoslavie, Belgrade : Medjunarodna Politika, 1966.

39. Selon Savezni Zavod za Statistiku SFRJ, op.cit., 1990, T.203-9.

40. Schrenk (Martin), Ardalan (Cyrus), El Tatawi (Nawal A.), Yugoslavia. Self Management and the Challenges of Development, Washington : World Bank, 1979.

41. OCDE, op.cit..

42. Lydall (Harold), op.cit.

43. Avec un taux d'utilisation des capacités à $60 \%$, contre $80 \%$ dans le monde occidental. Voir Zečević (P.), «Društveno-ekonomski razvoj 1970-1990 », Jugoslovenski pregled, (11-12), 1990, p. 283.

Avec un taux d'utilisation des capacités à $60 \%$, contre $80 \%$ dans le monde occidental (OCDE, op.cit).

44. Savezni Zavod za Statistiku SFRJ, op.cit., 1990, T 201-12.

45. En consommant le quart des ressources du fonds fédéral pour les régions les moins développées. (Djurdjević I., 1987)

46. Savezni Zavod za Statistiku SFRJ, op.cit., 1980 ; Savezni Zavod za Statistiku SFRJ, op.cit., 1990, T 204-1. 
47. Malgré des entrées en retraites croissantes, 171266 sur la période, soit dans les conditions du pays, autant de personnes recrutées (Savezni Zavod za Statistiku SFRJ, op.cit., 1990, T 201-32).

48. Trklja (Milivoje), «Sredstva društvenih delatnosti 1975-1984», Jugoslovenski pregled, (4), 1986.

49. JNA, art.cit., pp. 490-491.

50. Quelques milliers de VW Golf, de bicyclettes UNIS et de clones de machines à écrire mécaniques Olympia ne justifiaient pas autant de salariés.

51. Bojičić (Vesna), "Bosnia and Herzegovina. An Extreme Case of Transition », in Bianchini (Stefano), Uvalić (Milica), The Balkans and the challenge of economic integration, Ravenna: Longo, 1996.

52. Peut-être explicables par le phénomène Agrokomerc, mis à part le classique suremploi, facile à pratiquer dans ce secteur.

53. Uvalić (Milica), op.cit. ; Dirlam (Joel B.), Plummer (James L.), An introduction to the Yugoslav economy, Columbus : Charles E. Merrill, 1973.

54. Woodward (Susan L.), op.cit.

55. Resterait à examiner s'il s'agissait d'exportations en devises convertibles (matières premières et produits semi-finis) ou de matériels industriels (et militaires) pour lesquels l'exportation se faisait en direction de pays à monnaie non convertible, voire de clearing.

56. Milivojević (Marko), op.cit.Selon le rapport de l'OCDE pour 1986 (OCDE, op.cit.), seulement $4 \%$ du Produit social (društveni proizvod) était consacré à la défense ; si la moitié en partait en BiH, cette dernière recevait un transfert de $2 \%$ de la richesse nationale, ce qui augmentait sa richesse de $10 \%$.

57. Le parallèle avec les Glavki soviétiques a pu être suggéré. Leur importance comme instance décisionnelle associée aux pouvoirs régionaux dans les errements des années 1980 laisse sceptique face à la thèse selon laquelle les organisations élémentaires (OOUR, OBTA) auraient été coupables de l'irrationalité ambiante.

58. OCDE, op.cit.

59. Il n'a pas été établi de traduction pour cette expression anglo-saxonne courante pour désigner le dépouillement (stripping) des actifs (assets), si fréquent dans les premières phases de transition post-socialiste.

60. Muhamedagić (Sulejman), Oruč (Mirsada), « Historical survey of iron and steel production in Bosnia and Herzegovina », Materiali in Tehnologia, 43, 2009 ;EBRD, "Feature story. Mittal's Bosnia deal signs confidence », 2006, sur www.ebrd.com/news/stories/2006/060111.html.

61. Il s'agit là de modes de décomptes qui empoisonnent les relations entre communautés en Macédoine, chacun comptant ses émigrés, parfois à long terme, comme des citoyens temporairement émigrés (Roux (Michel), Les Albanais en Yougoslavie, minorité nationale, territoire et développement, Paris : MSH, 1992).

62. Istraživački Dokumentacioni Centar/Norvegian Ministry of Foreign Affairs, op.cit.

63. World Bank, Bosnia and Herzegovina. Challenges and Directions for Reform. A Public Expenditure and Institutional Review, Document of the world Bank, report (66253-BA), 2012.

La Banque mondiale, dans Migration and remittances Factbook 2008, compte 1471594 émigrés, soit $37,7 \%$ de la population.

64. En fait, la RS est en légère chute démographique (-3 370 en 2009), compensée par une légère expansion en $\mathrm{FBiH}(+3$ 342).

65. Il n'a pas été possible d'identifier précisément toutes les données pour le district de Brčko, ce qui entraîne un léger biais, mais sans influence structurelle d'interprétation des ressemblances et dissemblances entre entités.

66. Encore une coquetterie de l'autogestion, les services à la personne étaient enregistrés, à la différence des comptabilités de type soviétique, mais sans aller jusqu'à l'alignement sur les « capitalistes ». 
67. Peračković (Krešimir), « Hrvatska u post-industrijsko doba », Društvena istraživanja, 20 (1), 2011.

68. Bredenkamp (Caryn), Gragnolati (Michele), Ramljak (Vedad), Enhancing Efficiency and Equity. Challenges and Reforms Opportunities Facing Health and Pension Systems in the Western Balkans, World Bank, 2008 (Research Paper n46706) (http://siteresources.worldbank.org/ HEALTHNUTRITIONANDPOPULATION/Resources/281627-1095698140167/ BredenkampEnhancingEfficiency\&Equity.pdf).

69. Le total $\mathrm{BiH}$ a été calculé par addition des chiffres pour la Fédération et pour la RS, il ne correspond pas aux chiffres globaux pour la $\mathrm{BiH}$, car il exclut le District de Brčko, dont l'intégration aurait été délicate.

70. Bosansko-hercegovačka Agencija za Statistiku, BiH u brojevima 2010, Sarajevo : BHAS, 2010 ; Republika Srpska, Republički Zavod za Statistiku, Ovoje Republika Srpska, 2010.

71. Un examen d'annuaire d'entreprises nous permet de douter de l'application stricte de ce report. Il semble que certaines entreprises, comme les électriciens de bâtiment ou poseurs d'équipements domestiques, préfèrent se faire classer comme entreprises productives.

72. Bosansko-hercegovačka Agencija za Statistiku, Industrijska Proizvodnja u 2007 godini, Prethodni PRODCOM rezultati, 2007.

73. VW a continué une coopération pour les équipements de véhicules avec Prevent, mais a arrêté sa production de véhicules complets.

74. Kaminski (Bartlomiej), Ng (Francis), «Bosnia and Herzegovina's surprising export performance ", World Bank PRWP (5187), 2010 ; Domazet (Anto), Influence of Global Economic Crisis on Bosnia and Herzegovina, Sarajevo: Ekonomski Fakultet $\mathrm{u}$ Sarajevu/ Regional Cooperation Council, 2009 (presentation .ppt sur rcc.int, 21/02/2012).

75. Par exemple, Vlada FBiH, « O finansijkoj konsolidaciji rudnika uglja u FBiH », 04/02/2008 sur www.fbihvlada.gov.ba/aktuelno.

76. Hiver 2012, problèmes de prix de fournitures de courant à la raffinerie d'aluminium de Mostar.

77. Services rendus principalement aux entreprises, en nomenclature française.

78. Bosansko-hercegovačka Agencija za Statistiku, op.cit., p. 19 (« Tržište rada »).

79. Peračković (Krešimir), art.cit.

80. Kaminski (Bartlomiej), Ng (Francis), art.cit.; World Bank, Agricultural Sector Policy Note for Bosnia and Herzegovina, report (57919-BA), 2010.

81. Cf. Bosansko-hercegovačka Agencija za Statistiku, op.cit., 2010, pp. 31-32.

82. L'auteur peut témoigner que dans le cadre de l'application des accords-cadre d'ohrid en ARYM, il était très nettement apparu une contradiction entre une demande des institutions internationales du développement de réduire les dépenses publiques d'administration et les exigences de bureaucratie aux fins de mise en application de l'accord.

83. Republika Srpska, Republički Zavod za Statistiku, op.cit., T 7.3.1; Federacija Bosne i Hercegovine, Federalni Zavod za Statistiku, Federacija Bosne i Herzegovine u brojkama 2010, 2010, T.9.5.

84. Pratiquement sans incidence sur l'investissement, hors quelques grosses privatisations, dont le secteur bancaire.

85. Radošević (Slavo), « Research and Development, Competitiveness and European Integration of South Eastern Europe », Europe Asia Studies, 61 (4), 2009.

86. Kaminski (Bartlomiej), Ng (Francis), art.cit.

87. Bosansko-hercegovačka Agencija za Statistiku, Zaposleni po djelatnostima, Sarajevo : BHAS (Tematski bilten, (12), 2009).

88. En rappelant que pratiquement la moitié de l'investissement part en bâtiments, ce qui correspond sans doute à l'importance du secteur commercial, et qui révèle par ailleurs une performance faible dans l'industrie. 
89. Halilbašić (Muamer), "Strane direktne investicije i konkurentnost Bosne i Hercegovine ", Forum Bosnae, (52), 2011 ; Centralna Banka Bosna i Herzegovina, Annual review, 2010.

90. EBRD, art.cit.

91. Bosansko-hercegovačka Agencija za Statistiku, op.cit., 2010.

92. Ce qui signifie en fait une amélioration de la situation sur le plan salarial (SGJ 1990, p. 414-415), ce qui confirme nos conclusions précédentes.

93. FAO, Country profiles, Bosnia and Herzegovina 2012 (www.fao.org); Bosansko-hercegovačka Agencija za Statistiku, Anketa o potrošnji domaćinstava u BiH 2007, 2007 (www.bhas.ba); Bosansko-hercegovačka Agencija za Statistiku, Anketa o potrošnji domaćinstava u BiH 2011, 2011.

94. World Bank, Protecting the Poor During the Global Crisis. BH Poverty Update, rapport n51847-BH du 12-07-2009.

95. Bosansko-hercegovačka Agencija za Statistiku, Bruto Domaći Proizvod, 2005-2010, Sarajevo : BHAS 2011.

96. Bosansko-hercegovačka Agencija za Statistiku, op.cit., 2010.

97. RS, «Plaća dovoljna samo za 44,7 \% potrošačke korpe », RSE, 26/02/2012.

98. World Bank, op.cit., 2012, p. 20.

99. Ces profits sont minimes, dans son budget 2010-2011, la FBiH escompte 240 millions KM de dividendes à percevoir pour un total de 1795 millions.

100. Ce qui met en évidence au passage la grande faiblesse des profits dans l'économie bosniaque : 2700 millions de KM amortissements déduits, en enlevant le revenu des agriculteurs et les loyers recalculés autoconsommés. La faible part des dividendes dans les recettes de la FBiH (550 millions KM au budget 2011) nous conforte dans notre approche)

101. Centralna Banka Bosna i Herzegovina, « Neosmatrana i siva ekonomija u BiH », (Mimeo), dec. 2008, pp. 27-39.

102. Tomaš (Rajko), Kriza i siva ekonomija u Bosni i Herzegovini, Sarajevo : CBBH, 2010. Le Pr Rajko Tomaš évalue à $26 \%$ du PIB l'économie grise en 2009, pour un revenu de 2600 millions de KM, ce qui constitue une somme proche de celle obtenue par les remises de l'étranger (Tomaš (Rajko), « Mogućnosti uticaja države na makroekronomsku stabilnost i ublažavanje posljedica ekonomske krize u Bosni u Hercegovini », in Bosna i Hercegovina - 2014 Gdje želimo stići ?, Sarajevo : Friedrich Ebert Stiftung, 2009).

103. Centralna Banka Bosna i Herzegovina, op.cit., 2010.

104. World Bank, Izvještaj o uskladjenosti standarda i kodeksa (ROSC). Procjena korporativnog upravljanja Bosna i Hercegovina, ROSC report, 2006.

105. World Bank, op.cit., 2012; Nikšić (Orhan), "Strukturni razlozi nemogućnosti bosanskohercevačkoga odgovora », Forum Bosnae, (47), 2009.

106. Mišić (Ranka), « 31\% radnika ne dobija redovnu plaću », RSE, 15/02/2012.

107. « Energoinvest u krizi », RSE, 23/03/2009.

108. Sans parler de la question quelque peu démagogique des indemnités des élus.

109. World Bank, op.cit., 2012, p. 26.

110. World Bank, op.cit., 2010.

111. Bosna i Herzegovina (2010), Vjeće Ministara - Direkcija za ekonomsko planiranje (DEP), Ekonomski Trendovi, 2010 et 2011.

112. D'autant plus qu'en $\mathrm{BiH}$, les sociétés de commerce extérieur socialistes n'avaient pas la même importance qu'en Serbie, où elles ont largement contribué au système en place de 1991 à 2000.

113. Davezies (Laurent), La République et ses Territoires, Paris : Seuil, 2008.

114. World Bank, op.cit., 2010.

115. En témoignage de la conscience de la nécessité d'une évolution contrôlée, les initiatives récentes de relance du secteur de l'armement (comme par hasard, toutes entités réunies), un secteur resté solidement étatique. 
116. Mahmutćehajić (Rusmir), « Energetika u mraku energetizma », Forum Bosnae, (52), 2011.

117. IMF, Country Economic Report, Bosnia and Herzegovina, 08-06-2008.

118. Vlada Federacije BiH, Strateški partneri za realizaciju novih elektroenergetskih objekata u FBiH, Sarajevo (mimeo), 2008 ; Ekonomska Politika, (1003), 2008.

119. Ici encore, des dirigeants influencés par le souvenir du plan Goelro, mais aussi par les longs séjours d'entreprises bosniaques dans des États pétroliers, et à la recherche de substituts qui perpétueraient le système redistributif.

120. El Katiri (Laura), Fattouh (Bassam), Segal (Paul), Anatomy of an Oil Based Welfare State. Rent Distribution in Kuwait, Research Paper sur www.alternatives (journal.net/vol/number1), 2011, consulté le 20/02/2012; Munson (Peter J.), «Beyond the Rentier State. The distributive State in the Arab World », Government in the Lab, 2011 (http://govinthelab.com/beyond-the-rentier-state, consulté le 22/02/2012) ; Basedau (Mathias), Lacher (Wolfram), «A paradox of Plenty? Rent Distribution and Political Stability in Oil States », GIGA Working paper, (21), April 2006 (sur www.giga-hamburg.de consulté le 22/02/2012); Kuru (Ahmet), "The Rentier State Model and Central Asian Studies. The Turkmen Case », Alternatives. Turkish Journal of International Relations, 1 (1), Spring 2002 ; Delacroix (Jacques), "The distributive State in the World system ", Studies in comparative international development, 15 (3), 1980.

121. Henni (Ahmed), Le Cheikh et le Patron, Alger : Office d'Éditions Universitaires, 1993.

122. Belal (Youssef), Le Cheikh et le Calife, Paris : ENS, 2011.

123. Au rebours de ce qu'attendent les investisseurs moyen-orientaux, et surtout turcs, qui se situent aux antipodes de l'esprit de rente: «Medjunarodni investitori traže konkretnije uslove poslovanja », RFE, 02/04/2010 (consulté sur www.slobodnaevropa.org le 12/04/2010).

\section{RÉSUMÉS}

Dix sept ans après la sortie de conflit, alors que les dispositions consécutives aux accords de Dayton ont pacifié le pays par une décentralisation territoriale étendue, y compris en matière économique, la Bosnie Herzégovine a rattrapé son niveau de développement économique relatif du début des années 1990, ce qui la place néanmoins tout en bas de l'échelle européenne, en compagnie de la Macédoine et de l'Albanie. Au temps yougoslave, le dynamisme économique était mesuré, comparé à la pression démographique. L'émigration interne et externe évacuait une part des personnes qui seraient autrement restées sans emploi. On constate que la désindustrialisation postérieure à 1992 a surtout touché des activités politiques comme les armements, autrefois liés au non alignement, laissant en place des activités fondées historiquement sur les ressources naturelles, tandis que la tertiarisation marchande n'a pas eu d'effet moteur, et que la décentralisation liée au régime de Dayton (entités et cantons) na en rien réduit en rien l'emploi dans l'administration. Les basses performances de l'industrie sont compensées par un Etat, souvent actionnaire, qui laisse se reconstituer un régime d'illiquidité revenant aux pratiques socialistes de contrainte budgétaire molle, et ce malgré une législation alignée sur les standards internationaux, un régime monétaire strictement contrôlé et un monitoring international sévère sur les budgets.

De fait, l'apparente récupération économique repose sur le cumul des chocs démographiques : la baisse de population répartit la richesse produite sur moins de têtes, et l'émigration solvabilise les populations (par les remises), mais aussi l'Etat (par la TVA sur les importations). Le niveau de 
consommation a considérablement cru par rapport au temps du régime socialiste, alors que la population au travail a diminué, et que les rémunérations sont loin de couvrir les dépenses des ménages. Il faut donc tenir compte des consommations en nature, du travail dissimulé, des remises de l'émigration, mais également de l'impact social des dépenses de l'Etat. Celui-ci contrôle assez largement les rémunérations dans les entreprises où il détient des participations, ainsi que dans le secteur non marchand, et maintient des aides sociales ciblées sur des clientèles sensibles.

Or, l'État, ce sont avant tout les deux entités, Fédération de Bosnie-Herzégovine et République serbe. Les pratiques économiques publiques dans les deux entités sont très peu différenciées, et leurs performances économiques diffèrent peu. On peut donc en conclure que les Etats identitaires qui se sont constitués à la suite de Dayton se sont donné un objectif principal de redistribution. Cette dernière joue avec les règles économiques pour stabiliser l'emploi, et joue avec les sources budgétaires pour rendre solvable un large spectre de population.

Fort opportunément pour les détenteurs du pouvoir, l'essence même des activités productives réside dans un patrimoine naturel. Il ne reste donc plus aux autorités qu'à rationaliser cette situation en déclarant que le pays est riche de sa nature. On retrouve alors la logique des États redistributeurs (en général dotés d'hydrocarbures ou de minerais à haute valeur). Or, fort opportunément, du côté de la Fédération de Bosnie-Herzégovine, on dispose de la référence des États moyen-orientaux ou des républiques rentières d'Asie centrale, et du côté de la République serbe, de la Russie tout aussi rentière. Ce type de référence permet de satisfaire les aspirations identitaires, à rebours des idéaux du socialisme yougoslave qui voulait mettre l'enjeu productif au cœur de l'identité.

Seventeen years since the end of the war, the pacific solution for Bosnia and Herzegovina, achieved through a high level of decentralization, did not give substantial results in the field of economics. Yet the country recovered its international ranking from the time of former Yugoslavia, but still remains at the low end of the European scale, together with Macedonia, Albania (countries which didn't suffer from war), and Kosovo. In the socialist past, development went on a measured pace, Bosnia never improved its ranking among the Republics, and the demographic pressure, though going towards demographic transition, was never met by jobs creation. Migration, mainly internal, but also international, alleviated the demographic burden.

Post 1992 deindustrialization hit mostly the local "political" plants, but also the federal firms integrated in the Yugoslav military industrial complex. Eventually, most of the surviving firms are linked to the historical trend of natural resources based industrial activity. Since there has been no pull development of the tertiary sector, the public non tradable sector remains overdeveloped.

The State remains a sizeable shareholder in industry, and therefore is able to bias economic constraints through practices which remind the soft budget constraints from the time of socialism, whatever be the legal, financial and monetary patterns introduced according to the international standards. Formal employment in existing firms is given priority, together with a minimal distribution of revenues.

Most of the apparent improvement of macro data concerning the country is the result of a demographic shock since 1996 : the war losses and emigration, the decreasing trends in natural demography improve the currency inflows as much as they improve the per capita data. By the way, the remittances consolidate the state revenue through the VAT on imports.

So, the paradox is that the level of consumption has been going better and better since 1996, contrary to the productive trends. We must then take in consideration other sources of revenue, beginning with the afore mentioned remittances, the generalized ownership of housing, an adverse trend towards urbanization linked with natural forms of consumption, moonlighting, But the distributive expenses of the State play an important role towards sensible sectors of 
population, absorbing the main part of the public budgets.

In the Bosnian case, State means before all the ethnic entities, and subsidiarily the Districts in the Federation of Bosna and Herzegovina. When we come to consider that so much alleged difference gives way to such a similarity in the development pattern, and in the State's economic commitment, we can induce that the main function of the entities is a protective one, by ways of the repartition of the revenue in order to keep the citizens support.

The main productive activities relying on natural resources, everybody is induced to believe that the country can live from nature, as a collective heritage. This conception seeks its references among the rentier States, mostly oil and gas productors, whose population is largely subsidized from the trading of the natural resources. Of course, Bosnia and Herzegovina is far from these references, but it happens that the Federation can refer to the Middle Eastern or Central Asian states, and the Serb republic can peep towards Russia, another oil rentier state.

So, identity aspirations can be fulfilled, in an adverse trend towards both the international expectations for an insertion in the dynamics of international development, and the former tasks of socialism which fixed economic development through productive activity as the main cement of national identity.

\section{AUTEUR}

\section{HUGUES SACHTER}

DR HDR

Chercheur associé au CLERSE (Université Lille 1) 